\title{
UNIFORM NULL-CONTROLLABILITY FOR THE ONE-DIMENSIONAL HEAT EQUATION WITH RAPIDLY OSCILLATING PERIODIC DENSITY
}

\author{
A. LÓPEZ ${ }^{1}$, E. ZUAZUA ${ }^{1}$ \\ Departamento de Matemática Aplicada, Universidad Complutense de Madrid, 28040 Madrid, Spain
}

Received 15 March 2001, revised 2 June 2001

ABSTRACT. - We consider the $1-d$ heat equation with rapidly oscillating periodic density in a bounded interval with Dirichlet boundary conditions. When the period tends to zero and the density weakly converges to its average we prove that the boundary controls converge to a control of the limit, constant coefficient heat equation when the density is $C^{2}$.

The proof is based on a control strategy in three steps in which: we first control the low frequencies of the system, we then let the system to evolve freeely and, finally, we control to zero the whole solution. We use the theory of real exponentials to analyze the low frequencies and Carleman inequalities to control the whole solution.

The result is in constrast with the divergent behavior of the null controls for the wave equation with rapidly oscillating coefficients.

(C) 2002 L'Association Publications de l'Institut Henri Poincaré. Published by Elsevier B.V. All rights reserved

RÉSUMÉ. - On considère l'équation de la chaleur $1-d$ avec densité périodique rapidement oscillante de classe $C^{2}$ dans un intervalle borné avec des conditions aux limites de Dirichlet. On démontre que, lorsque la période tend vers zéro et donc la densité converge faiblement vers sa moyenne, les contrôles convergent vers un contrôle pour l'équation de la chaleur limite, à coefficients constants.

Notre construction se fait en trois étapes. Dans la première nous contrôlons uniformement les bases fréquences. Dans une deuxième étape nous laissons les solutions décroitre sans contrôle. Finalement, nous appliquons un contrôle qui ramène les solutions à zéro. La preuve combine la théorie des sommes d'exponentielles réeles pour analyser les basses fréquences et les inégalités de Carleman pour ramener les solutions à zéro.

Ce résultat est à comparer avec ceux établis dans le cadre de l'équation des ondes à coefficients rapidement oscillants. Dans ce dernier cas on sait que les contrôles divergent lorsque la période tend vers zéro.

(C) 2002 L'Association Publications de l'Institut Henri Poincaré. Published by Elsevier B.V. All rights reserved

E-mail addresses: bantonio@sunma4.mat.ucm.es (A. López), enrique.zuazua@uam.es (E. Zuazua).

${ }^{1}$ Supported by grant PB96-0663 of the DGES (Spain) and the EU TMR Project "Homogenization and Multiple Scales". 


\section{Introduction and main results}

Let $\rho \in W^{2, \infty}(\mathbb{R})$ be a periodic function satisfying

$$
0<\rho_{m} \leqslant \rho(x) \leqslant \rho_{M}<+\infty,
$$

for all $x \in \mathbb{R}$. We recall that $W^{2, \infty}(\mathbb{R})$ is the Sobolev space of $L^{\infty}(\mathbb{R})$ functions whose derivatives up to the second order are in $L^{\infty}(\mathbb{R})$.

Without lost of generality we may assume that $\rho$ is periodic of period 1 . We denote by $\bar{\rho}$ its average

$$
\bar{\rho}=\int_{0}^{1} \rho(x) \mathrm{d} x .
$$

This paper is devoted to analyze the null-controllability of the following heat equation with oscillating density

$$
\begin{cases}\rho\left(\frac{x}{\varepsilon}\right) u_{t}^{\varepsilon}-u_{x x}^{\varepsilon}=0, & 0<x<1,0<t<T, \\ u^{\varepsilon}(0, t)=0, \quad u^{\varepsilon}(1, t)=f^{\varepsilon}(t), & 0<t<T, \\ u^{\varepsilon}(x, 0)=u_{0}(x), & 0<x<1 .\end{cases}
$$

The results in [8] and [9] show that for any $T>0, \varepsilon \in(0,1)$ and $u_{0} \in L^{2}(0,1)$ there exists a control $f^{\varepsilon} \in L^{2}(0, T)$ such that the solution of (3) verifies

$$
u^{\varepsilon}(x, T)=0, \quad \text { for all } 0<x<1 .
$$

In fact there exists, for all $T>0$ and $\varepsilon \in(0,1)$, a positive constant $C(\varepsilon, T)$ such that

$$
\left\|f^{\varepsilon}\right\|_{L^{2}(0, T)} \leqslant C(\varepsilon, T)\left\|u_{0}\right\|_{L^{2}(0,1)},
$$

for all $u_{0} \in L^{2}(0,1)$.

The main goal of this paper is to show that $C(\varepsilon, T)$ remains bounded as $\varepsilon \rightarrow 0$.

The formal limit as $\varepsilon \rightarrow 0$ of (3) is the averaged system

$$
\begin{cases}\bar{\rho} u_{t}-u_{x x}=0, & 0<x<1,0<t<T, \\ u(0, t)=0, \quad u(1, t)=f(t), & 0<t<T, \\ u(x, 0)=u_{0}(x), & 0<x<1 .\end{cases}
$$

The limit system (6) is also null-controllable.

Therefore, it is natural to analyze whether the constant $C(\varepsilon, T)$ in (5) remains bounded as $\varepsilon \rightarrow 0$ and if the control $f$ of system (6) is actually the limit of the controls $f^{\varepsilon}$ of systems (3) as $\varepsilon \rightarrow 0$.

These questions are indeed natural to be addressed from the point of view of homogenization theory. Indeed, the goal in homogenization is to analyze the limit behavior of a system in the presence of rapidly oscillating coefficients and to obtain the effective limit system. This is relevant from a computational point of view since this limit process may allow to replace the system with rapidly oscillating coefficients by the 
homogenized one when performing the computations. In the context of controllability the same phylosophy applies and therefore it is natural to analyze whether the control of (3) converge to the control of (6). When this is true, for practical purpouses, one can compute the control $f$ of the limit system (6). This is a much simpler task since the system in (6) and corresponding adjoint system have constant coefficients.

When addressing this question we were also motivated by the negative results in [1] that show that, in the context of the wave equation, the observability constant blows-up exponentially as $\varepsilon \rightarrow 0$ (see also [2]-[4]).

As we shall see, in the context of the heat equation under consideration the constants $C(\varepsilon, T)$ in (5) remain bounded as $\varepsilon \rightarrow 0$. This shows that the dissipativity of the heat equation compensates the spectral pathologies leading to the blow-up of the observability constant in the context of the wave equation. Theorem 1.1 below shows that the situation is completely different for the heat equation.

The main result of this paper is the following:

THEOREM 1.1. - Assume that $\rho \in W^{2, \infty}(\mathbb{R})$ is a periodic function of period 1 satisfying (1). Let $T>0$. Then, for any $u_{0} \in L^{2}(0,1)$ and $\varepsilon \in(0,1)$ there exists a control $f^{\varepsilon} \in L^{2}(0, T)$ such that the solution $u^{\varepsilon}$ of (3) satisfies (4). Moreover, there exists a constant $C(T)$, independent of $\varepsilon \in(0,1)$, such that

$$
\left\|f^{\varepsilon}\right\|_{L^{2}(0, T)} \leqslant C(T)\left\|u_{0}\right\|_{L^{2}(0,1)} .
$$

Finally, for any $u_{0} \in L^{2}(0,1)$ fixed, there exists a sequence of controls $\left\{f^{\varepsilon}\right\}$ for system (3) such that

$$
\left\{f^{\varepsilon}\right\} \rightarrow f \text { strongly in } L^{2}(0, T) \text { as } \varepsilon \rightarrow 0,
$$

$f$ being a control of the limit problem (6), so that the solution of (6) satifies

$$
u(x, T)=0, \quad \text { for all } 0<x<1 .
$$

Moreover, the sequence $\left\{u^{\varepsilon}\right\}$ of solutions of (3) are bounded in $L^{\infty}\left(0, T ; H^{-s}(0,1)\right)$ and, in fact,

$$
\left\{u^{\varepsilon}\right\} \rightarrow \text { uweakly-*in } L^{\infty}\left(0, T ; H^{-s}(0,1)\right) \quad \text { as } \varepsilon \rightarrow 0,
$$

for all $s>\frac{3}{2}$.

The proof of Theorem 1.1 is based on a control strategy in three steps inspired from [14]. The same control strategy has been used before in [17] and [18]. Roughly, the proof is as follows. We divide the time interval $[0, T]$ in three subintervals: $I_{1}=\left[0, \frac{T}{3}\right]$, $I_{2}=\left[\frac{T}{3}, \frac{2 T}{3}\right]$ and $I_{3}=\left[\frac{2 T}{3}, T\right]$. In the first time interval, $I_{1}$, we control to zero the projection of the solution over a suitable subspace containing only sufficiently low frequencies. In the second time interval, $I_{2}$, we let the system to evolve freely without control. In this way the projection of the solution of (3) over the low frequencies remains at rest and, due to the strong dissipativity of system (3) in its high frequencies, the size of the solution at time $t=2 T / 3$ becomes exponentially small, i.e. of the order of $\mathrm{e}^{-c / \varepsilon^{2}}$ for a suitable constant $c>0$, as $\varepsilon \rightarrow 0$. Finally, in the interval $I_{3}$ we apply a control driving the whole solution to zero. 
Using Carleman estimates it can be proved that the control needed in the third interval is at most of the order of $\mathrm{e}^{C / \varepsilon^{4 / 3}}$, for a suitable constant $C>0$, with respect to the solution at time $t=2 T / 3$. However, since, in view of the analysis made in the interval $I_{2}$, the norm of the solution, in the absence of control, at time $t=2 T / 3$ decays by a multiplicative factor of the order of $\mathrm{e}^{-C / \varepsilon^{2}}$, these two phenomena compensate and the control needed in the third interval $I_{3}$ turns out to be uniformly bounded, and even exponentially small as $\varepsilon \rightarrow 0$.

As an immediate corollary, by duality, it can be shown that the following uniform observability result holds for the adjoint system

$$
\begin{cases}\rho\left(\frac{x}{\varepsilon}\right) \phi_{t}^{\varepsilon}+\phi_{x x}^{\varepsilon}=0, & 0<x<1,0<t<T, \\ \phi^{\varepsilon}(0, t)=\phi^{\varepsilon}(1, t)=0, & 0<t<T, \\ \phi^{\varepsilon}(x, T)=\phi^{0}(x), & 0<x<1 .\end{cases}
$$

THEOREM 1.2. - Under the assumptions of Theorem 1.1, there exists a constant $C>0$, independent of $0<\varepsilon<1$, such that

$$
\left\|\phi^{\varepsilon}(\cdot, 0)\right\|_{L^{2}(0,1)}^{2} \leqslant C \int_{0}^{T}\left|\phi_{x}^{\varepsilon}(1, t)\right|^{2} \mathrm{~d} t,
$$

holds for every $\phi^{\varepsilon}$ solution of (11) with $\phi^{0} \in L^{2}(0,1)$.

The rest of the paper is organized as follows. In Section 2 we describe in detail the iterative method we use to prove the uniform boundedness of the constant $C(\varepsilon, T)$ in (5). In Section 3 we obtain a partial uniform controllability result. Section 4 is devoted to obtain a global but non-uniform controllability result on the solutions of system (3). Both controllability results are needed in our iterative method. In Section 5 we complete the proof of the first part of Theorem 1.1 and in Section 6 we develop the limit process. Finally in Section 7 we comment on some possible extensions of the results of this paper.

\section{Description of the "three-steps" controllability method}

In this section we describe in detail the "three-steps" method outlined in the introduction allowing to prove the null controllability of system (3) in such a way that, with a further careful analysis, the controls may be shown to be uniformly bounded.

In order to develop this method we need to decompose the spectrum of system (3) into two parts that we will refer to as low and high frequencies of system (3) from now.

\subsection{Spectral analysis}

Consider the eigenvalue problem associated to system (3):

$$
\left\{\begin{array}{l}
\lambda \rho\left(\frac{x}{\varepsilon}\right) \varphi^{\varepsilon}+\varphi_{x x}^{\varepsilon}=0, \quad 0<x<1 \\
\varphi^{\varepsilon}(0)=\varphi^{\varepsilon}(1)=0
\end{array}\right.
$$


For each $\varepsilon \in(0,1)$ there exists a sequence of eigenvalues

$$
0<\lambda_{1}^{\varepsilon}<\lambda_{2}^{\varepsilon}<\cdots<\lambda_{n}^{\varepsilon}<\cdots \rightarrow+\infty
$$

and a sequence of associated eigenfunctions $\left(\varphi_{n}^{\varepsilon}\right)_{n \in \mathbb{N}}$ which can be chosen to constitute an orthonormal basis in $L^{2}(0,1)$ with the norm

$$
\|\varphi\|_{L^{2}(0,1)}^{2}=\int_{0}^{1} \rho\left(\frac{x}{\varepsilon}\right)|\varphi(x)|^{2} \mathrm{~d} x .
$$

The eigenfunctions are also orthogonal in $H_{0}^{1}(0,1)$ and satisfy

$$
\int_{0}^{1}\left|\left(\varphi_{k}^{\varepsilon}\right)_{x}(x)\right|^{2} \mathrm{~d} x=\lambda_{k}^{\varepsilon} \int_{0}^{1} \rho\left(\frac{x}{\varepsilon}\right)\left|\varphi_{k}^{\varepsilon}(x)\right|^{2} \mathrm{~d} x=\lambda_{k}^{\varepsilon} .
$$

On the other hand, using the characterization of the eigenvalues $\lambda_{k}^{\varepsilon}$ given by the Rayleigh quotient:

$$
\lambda_{k}^{\varepsilon}=\max _{\operatorname{dim} E=k} \min _{\varphi \in E} \frac{\int_{0}^{1}\left|\varphi_{x}\right|^{2} \mathrm{~d} x}{\int_{0}^{1} \rho\left(\frac{x}{\varepsilon}\right)|\varphi|^{2} \mathrm{~d} x}
$$

we deduce that

$$
\frac{k^{2} \pi^{2}}{\rho_{M}} \leqslant \lambda_{k}^{\varepsilon} \leqslant \frac{k^{2} \pi^{2}}{\rho_{m}} .
$$

Let us recall the following result from [4]:

Proposition 2.1. - Assume that $\rho \in L^{\infty}(\mathbb{R})$ is a periodic function satisfying (1). Given $\delta>0$, there exists a constant $C(\delta)$, independent of $\varepsilon \in(0,1)$, such that

$$
\sqrt{\lambda_{k+1}^{\varepsilon}}-\sqrt{\lambda_{k}^{\varepsilon}} \geqslant \frac{\pi}{\sqrt{\bar{\rho}}}-\delta
$$

for all $k \leqslant C(\delta) / \varepsilon$.

Futhermore, there exist positive constants $C_{1}, C_{2}$ and $C_{3}$, independent of $\varepsilon \in(0,1)$, such that

$$
C_{1}\left|\left(\varphi_{k}^{\varepsilon}\right)_{x}(1)\right|^{2} \leqslant \int_{0}^{1}\left|\left(\varphi_{k}^{\varepsilon}\right)_{x}(x)\right|^{2} \mathrm{~d} x \leqslant C_{2}\left|\left(\varphi_{k}^{\varepsilon}\right)_{x}(1)\right|^{2},
$$

for all $k \leqslant C_{3} / \varepsilon$.

Remark 2.1. - Note that (20) and (16) imply that

$$
\frac{\lambda_{k}^{\varepsilon}}{C_{2}} \leqslant\left|\left(\varphi_{k}^{\varepsilon}\right)_{x}(1)\right|^{2} \leqslant \frac{\lambda_{k}^{\varepsilon}}{C_{1}}
$$

for all $k \leqslant C_{3} / \varepsilon$. 
On the other hand, taking $\delta=\pi /(2 \sqrt{\bar{\rho}})$ in Proposition 2.1, we have

$$
\sqrt{\lambda_{k+1}^{\varepsilon}}-\sqrt{\lambda_{k}^{\varepsilon}} \geqslant \frac{\pi}{2 \sqrt{\rho}}
$$

for $k \leqslant C(\pi /(2 \sqrt{\bar{\rho}})) / \varepsilon$.

Then (21) and (22) are valid for all $k \leqslant\left[D \varepsilon^{-1}\right]$ with

$$
D=\min \left\{C_{3}, C\left(\frac{\pi}{2 \sqrt{\bar{\rho}}}\right)\right\} .
$$

Here and in the sequel [.] denotes the integer part.

In view of Proposition 2.1 and Remark 2.1 we introduce the space

$$
H_{k \leqslant\left[D \varepsilon^{-1}\right]}=\left\{u \in L^{2}(0,1): u=\sum_{k \leqslant\left[D \varepsilon^{-1}\right]} a_{k} \varphi_{k}^{\varepsilon}(x)\right\},
$$

where $a_{k}$ are real numbers and $D$ is as in (23). $H_{k \leqslant\left[D \varepsilon^{-1}\right]}$ is simply the subspace of $L^{2}(0,1)$ generated by the first $\left[D \varepsilon^{-1}\right]$ eigenfunctions. We will call these eigenfunctions the low frequencies, and so $H_{k \leqslant\left[D \varepsilon^{-1}\right]}$ will be the space generated by the low frequencies. Note that $H_{k \leqslant\left[D \varepsilon^{-1}\right]}$ is a finite-dimensional space but its dimension increases as $\varepsilon$ decreases. This fact will play a key role in our proof. Given $u \in L^{2}(0,1)$ we will denote by $\Pi_{k \leqslant\left[D \varepsilon^{-1}\right]} u$ the orthogonal projection over $H_{k \leqslant\left[D \varepsilon^{-1}\right]}$.

\subsection{The control strategy}

We are now in conditions to decribe precisely our control strategy.

Given $T>0$ we divide the time interval in three subintervals

$$
[0, T]=I_{1} \cup I_{2} \cup I_{3},
$$

with $I_{1}=\left[0, \frac{T}{3}\right], I_{2}=\left[\frac{T}{3}, \frac{2 T}{3}\right]$ and $I_{3}=\left[\frac{2 T}{3}, T\right]$.

Given an initial datum $u_{0} \in L^{2}(0,1)$ to be controlled we proceed as follows:

- First step. In the first time interval $I_{1}$ we drive to zero the low frequencies, i.e. the projection over the space $H_{k \leqslant\left[D \varepsilon^{-1}\right]}$ of the solution. In other words, we introduce a control $f_{1}^{\varepsilon} \subset L^{2}(0, T / 3)$ such that the solution of

$$
\begin{cases}\rho\left(\frac{x}{\varepsilon}\right) u_{t}^{\varepsilon}-u_{x x}^{\varepsilon}=0, & 0<x<1,0<t<\frac{T}{3}, \\ u^{\varepsilon}(0, t)=0, \quad u^{\varepsilon}(1, t)=f_{1}^{\varepsilon}(t), & 0<t<\frac{T}{3}, \\ u^{\varepsilon}(x, 0)=u_{0}(x), & 0<x<1,\end{cases}
$$

satisfies

$$
\Pi_{k \leqslant\left[D \varepsilon^{-1}\right]} u^{\varepsilon}\left(\frac{T}{3}\right)=0 .
$$

As we will see, this can be done uniformly on $\varepsilon \in(0,1)$. Here and in the sequel we use the abridged notation $u^{\varepsilon}(t)$ to denote the $x$-dependent function $u^{\varepsilon}(x, t)$. More precisely, 
we will prove the existence of a positive constant $C>0$, independent of $\varepsilon \in(0,1)$, such that

$$
\left\|f_{1}^{\mathcal{E}}\right\|_{L^{2}\left(0, \frac{T}{3}\right)} \leqslant C\left\|u_{0}\right\|_{L^{2}(0,1)},
$$

for all $u_{0} \in L^{2}(0,1)$ and $\varepsilon \in(0,1)$. This result will be proved using classical results of nonharmonic Fourier series and Proposition 2.1.

In view of the uniform bound (28) of the control we shall also show that

$$
\left\|u^{\varepsilon}\left(\frac{T}{3}\right)\right\|_{L^{2}(0,1)} \leqslant C\left\|u_{0}\right\|_{L^{2}(0,1)} .
$$

We denote by $v_{0}^{\varepsilon}$ the solution obtained at the end of the first time interval $I_{1}$, i.e.

$$
v_{0}^{\varepsilon}=u^{\varepsilon}\left(\frac{T}{3}\right)
$$

- Second step. In the second time interval $I_{2}$ we let the equation to evolve freely. In other words, we solve

$$
\begin{cases}\rho\left(\frac{x}{\varepsilon}\right) u_{t}^{\varepsilon}-u_{x x}^{\varepsilon}=0, & 0<x<1, \frac{T}{3}<t<\frac{2 T}{3}, \\ u^{\varepsilon}(0, t)=u^{\varepsilon}(1, t)=0, & \frac{T}{3}<t<\frac{2 T}{3}, \\ u^{\varepsilon}\left(x, \frac{T}{3}\right)=v_{0}^{\varepsilon}, & 0<x<1 .\end{cases}
$$

Taking (24) into account it is easy to see that the solution of (31) verifies

$$
\Pi_{k \leqslant\left[D \varepsilon^{-1}\right]} u^{\varepsilon}(t)=0, \quad \text { for all } \frac{T}{3} \leqslant t \leqslant \frac{2 T}{3} .
$$

Futhermore, we will see that there exists a positive constant $C_{1}$ such that

$$
\left\|u^{\varepsilon}(\cdot, t)\right\|_{L^{2}(0,1)} \leqslant C_{1} \mathrm{e}^{-\lambda_{\left[D \varepsilon^{-1}\right]}^{\varepsilon}\left(t-\frac{T}{3}\right)}\left\|v_{0}^{\varepsilon}\right\|_{L^{2}(0,1)},
$$

for all $T / 3 \leqslant t \leqslant 2 T / 3$. In particular, for $t=2 T / 3$, taking (18) into account, we deduce that

$$
\left\|u^{\varepsilon}\left(\cdot, \frac{2 T}{3}\right)\right\|_{L^{2}(0,1)} \leqslant C_{1} \mathrm{e}^{-C_{2} / \varepsilon^{2}}\left\|v_{0}^{\varepsilon}\right\|_{L^{2}(0,1)},
$$

where the positive constants $C_{1}$ and $C_{2}$ do not depend on $\varepsilon \in(0,1)$.

Therefore, at the end of the second step, i.e. at time $t=2 T / 3$, we obtain a state $u^{\varepsilon}(\cdot, 2 T / 3)$ with an exponentially small (as $\varepsilon \rightarrow 0$ ) norm. We denote by $w_{0}^{\varepsilon}$ the solution at time $t=2 T / 3$, i.e.

$$
w_{0}^{\varepsilon}=u^{\varepsilon}\left(\frac{2 T}{3}\right)
$$


- Third step. In the last step we control the whole solution to zero. According to [11] there exists $f_{2}^{\varepsilon} \in L^{2}(2 T / 3, T)$ such that the solution of

$$
\begin{cases}\rho\left(\frac{x}{\varepsilon}\right) u_{t}^{\varepsilon}-u_{x x}^{\varepsilon}=0, & 0<x<1, \frac{2 T}{3}<t<T, \\ u^{\varepsilon}(0, t)=0, \quad u^{\varepsilon}(1, t)=f_{2}^{\varepsilon}(t), & \frac{2 T}{3}<t<T, \\ u^{\varepsilon}\left(x, \frac{2 T}{3}\right)=w_{0}^{\varepsilon}, & 0<x<1,\end{cases}
$$

satisfies

$$
u^{\varepsilon}(T)=0 .
$$

As we will prove in Section 4 using Carleman estimates, there exist positive constants $C_{1}$ and $C_{2}$ such that

$$
\left\|f_{2}^{\varepsilon}\right\|_{L^{2}\left(\frac{2 T}{3}, T\right)} \leqslant C_{1} \mathrm{e}^{C_{2} / \varepsilon^{4 / 3}}\left\|w_{0}^{\varepsilon}\right\|_{L^{2}(0,1)},
$$

for all $\varepsilon \in(0,1)$. The constants $C_{1}$ and $C_{2}$ in (38) depend on $T$ but they are independent of $\varepsilon \in(0,1)$.

Combining (29), (34) and (38) and taking (30) and (35) into account we get

$$
\left\|f_{2}^{\varepsilon}\right\|_{L^{2}\left(\frac{2 T}{3}, T\right)} \leqslant C_{1} \mathrm{e}^{C_{2} / \varepsilon^{4 / 3}} \mathrm{e}^{-C_{3} / \varepsilon^{2}}\left\|u_{0}\right\|_{L^{2}(0,1)},
$$

where the constants $C_{1}, C_{2}$ and $C_{3}$ are all of them independent of $\varepsilon \in(0,1)$.

Conclusion: Putting all these results together we conclude that the control

$$
f^{\varepsilon}= \begin{cases}f_{1}^{\varepsilon} & \text { in }\left[0, \frac{T}{3}\right] \\ 0 & \text { in }\left[\frac{T}{3}, \frac{2 T}{3}\right] \\ f_{2}^{\varepsilon} & \text { in }\left[\frac{2 T}{3}, T\right]\end{cases}
$$

is such that

$$
\left\|f^{\varepsilon}\right\|_{L^{2}(0, T)} \leqslant C\left\|u_{0}\right\|_{L^{2}(0,1)}
$$

for all $u_{0} \in L^{2}(0,1)$, with a constant $C>0$ independent of $0<\varepsilon<1$ and moreover the solution of (3) satisfies (4), as we wanted to prove.

Note also that the control $f^{\varepsilon}$ given by (40) is concentrated on the intervals $\left[0, \frac{T}{3}\right]$ and $\left[\frac{2 T}{3}, T\right]$ and that its restriction to the last interval $\left[\frac{2 T}{3}, T\right]$ is exponentially small (see (39)) with respect to $\varepsilon \rightarrow 0$.

The following two sections are devoted to rigorously prove the results stated in each of these three steps.

\section{Uniform controllability of the "low frequencies"}

The main result of this section is as follows:

THEOREM 3.1. - Assume that $\rho \in L^{\infty}(\mathbb{R})$ is a periodic function satisfying (1). Let $T>0$. Then, for any $u_{0} \in L^{2}(0,1)$ there exists a control $f^{\varepsilon} \in L^{2}(0, T)$ such that the solution of (3) satisfies

$$
\Pi_{k \leqslant\left[D \varepsilon^{-1}\right]} u^{\varepsilon}(T)=0 .
$$


Moreover, there exists a constant $C>0$, depending on $T$ but independent of $\varepsilon \in(0,1)$, such that

$$
\left\|f^{\varepsilon}\right\|_{L^{2}(0, T)} \leqslant C\left\|u_{0}\right\|_{L^{2}(0,1)}
$$

for all $u_{0} \in L^{2}(0,1)$.

Proof. - By duality, the proof of the controllability result of this theorem may be reduced to prove the following observability result:

Proposition 3.1.-Given any $T>0$, there exists a positive constant $C(T)$, independent of $\varepsilon \in(0,1)$, such that

$$
\int_{0}^{T}\left|\phi_{x}^{\varepsilon}(1, t)\right|^{2} \mathrm{~d} t \geqslant C(T)\left\|\phi^{\varepsilon}(\cdot, 0)\right\|_{L^{2}(0,1)}^{2}
$$

for any solution of system (11) with $\phi^{0} \in H_{k \leqslant\left[D \varepsilon^{-1}\right]}$.

Before getting into the proof of Proposition 3.1 let us show why it implies the result in Theorem 3.1.

For any $\varepsilon>0$ and $\delta>0$, following [6], we consider the funtional

$$
J_{\varepsilon, \delta}\left(\phi^{0}\right)=\frac{1}{2} \int_{0}^{T}\left|\phi_{x}^{\varepsilon}(1, t)\right|^{2} \mathrm{~d} t+\delta\left\|\phi^{0}\right\|_{H_{0}^{1}(0,1)}^{2}-\int_{0}^{1} \rho\left(\frac{x}{\varepsilon}\right) u_{0}(x) \phi^{\varepsilon}(x, 0) \mathrm{d} x .
$$

This funtional is continous and convex in $H_{0}^{1}(0,1)$. We note in particular that it is well defined in $H_{0}^{1}(0,1)$ since the solution $\phi^{\varepsilon}(x, t)$ of $(11)$ belongs to $L^{2}\left(0, T ; H^{2} \cap\right.$ $\left.H_{0}^{1}(0,1)\right)$ and therefore $\phi_{x}^{\varepsilon}(1, t) \in L^{2}(0, T)$. Moreover, according to Proposition 3.1, it is uniformly coercive in the subspace $V_{k \leqslant\left[D \varepsilon^{-1}\right]}$ of $H_{0}^{1}(0,1)$ generated by the eigenfunctions $\varphi_{k}^{\varepsilon}$ of 13 with $k \leqslant\left[D \varepsilon^{-1}\right]$.

Therefore, $J_{\varepsilon, \delta}\left(\phi^{0}\right)$ admits a unique minimizer in $V_{k \leqslant\left[D \varepsilon^{-1}\right]}$. It is easy to see that the control $f^{\varepsilon, \delta}=\widehat{\phi}_{x}^{\varepsilon, \delta}(1, t)$, where $\widehat{\phi}^{\varepsilon, \delta}(x, t)$ is the solution of (11) with the minimizer $\widehat{\phi}_{0}^{\varepsilon, \delta}(x)$ of $J_{\varepsilon, \delta}$ in $V_{k \leqslant\left[D \varepsilon^{-1}\right]}$ as initial data, is such that the solution $u^{\varepsilon, \delta}(x, t)$ of (3) satisfies

$$
\left\|u^{\varepsilon, \delta}(T)\right\|_{H^{-1}(0,1)} \leqslant \delta .
$$

Moreover, according to the uniform inequatily (44), it is easy to see that the controls $\left\{f^{\varepsilon, \delta}\right\}$ are uniformly bounded in $L^{2}(0, T)$ with respect to $\varepsilon \in(0,1)$ and $\delta \in(0,1)$.

Passing to the limit as $\delta \rightarrow 0$, with $\varepsilon>0$ fixed, we obtain a control $f^{\varepsilon}$ for system (3) such that, according to (45), the solution $u^{\varepsilon}$ of (3) satisfies

$$
u^{\varepsilon}(T)=0 .
$$

Moreover, the controls $\left\{f^{\varepsilon}\right\}$ remain also uniformly bounded in $L^{2}(0, T)$ with respect to $\varepsilon \in(0,1)$. 
Proof of Proposition 3.1. - By (24),

$$
\phi^{0}(x)=\sum_{k \leqslant\left[D \varepsilon^{-1}\right]} a_{k} \varphi_{k}^{\varepsilon}(x) .
$$

We develop the solution of (11) in Fourier series,

$$
\phi^{\varepsilon}(x, t)=\sum_{k \leqslant\left[D \varepsilon^{-1}\right]} a_{k} \mathrm{e}^{-\lambda_{k}^{\varepsilon}(T-t)} \varphi_{k}^{\varepsilon}(x) .
$$

In view of the orthogonality of the eigenfunctions $\left\{\varphi_{k}^{\varepsilon}\right\}$ in the space $L^{2}(0,1)$ with weight $\rho\left(\frac{x}{\varepsilon}\right)$ we have

$$
\begin{aligned}
\left(\frac{1}{\rho_{M}}\right)^{2} \sum_{k \leqslant\left[D \varepsilon^{-1}\right]}\left|a_{k}\right|^{2} \mathrm{e}^{-2 \lambda_{k}^{\varepsilon} T} \mathrm{~d} x & \leqslant \int_{0}^{1}\left|\sum_{k \leqslant\left[D \varepsilon^{-1}\right]} a_{k} \mathrm{e}^{-\lambda_{k}^{\varepsilon} T} \varphi_{k}^{\varepsilon}(x)\right|^{2} \mathrm{~d} x \\
& \leqslant\left(\frac{1}{\rho_{m}}\right)^{2} \sum_{k \leqslant\left[D \varepsilon^{-1}\right]}\left|a_{k}\right|^{2} \mathrm{e}^{-2 \lambda_{k}^{\varepsilon} T} \mathrm{~d} x .
\end{aligned}
$$

Thus inequatily (44) is equivalent to

$$
\int_{0}^{T}\left|\sum_{k \leqslant\left[D \varepsilon^{-1}\right]} a_{k} \mathrm{e}^{-\lambda_{k}^{\varepsilon}(T-t)}\left(\varphi_{k}^{\varepsilon}\right)_{x}(1)\right|^{2} \mathrm{~d} t \geqslant C(T) \sum_{k \leqslant\left[D \varepsilon^{-1}\right]}\left|a_{k}\right|^{2} \mathrm{e}^{-2 \lambda_{k}^{\varepsilon} T} .
$$

In view of (21), in order to prove (49) it is sufficient to show that

$$
\int_{0}^{T}\left|\sum_{k \leqslant\left[D \varepsilon^{-1}\right]} a_{k} \mathrm{e}^{-\lambda_{k}^{\varepsilon} t}\right|^{2} \mathrm{~d} t \geqslant C(T) \sum_{k \leqslant\left[D \varepsilon^{-1}\right]} \frac{\left|a_{k}\right|^{2}}{\lambda_{k}^{\varepsilon}} \mathrm{e}^{-2 \lambda_{k}^{\varepsilon} T}
$$

for every sequence $\left\{a_{k}\right\} \in l^{2}$. Inequality (50) may be obtained as a consequence of classical results on series of real exponentials that we describe now.

Given $\xi>0$ and a decreasing function $N:(0,+\infty) \rightarrow \mathbb{N}$, such that $N(\delta) \rightarrow+\infty$ as $\delta \rightarrow 0$, following [9], we introduce the class $\mathcal{L}(\xi, N)$ of increasing sequences of positive real numbers $\left\{\mu_{j}\right\}_{j} \geqslant 1$ such that:

$$
\begin{gathered}
\mu_{1} \geqslant \xi>0, \\
\mu_{j+1}-\mu_{j} \geqslant \xi>0, \quad \text { for all } j \geqslant 1,
\end{gathered}
$$

and

$$
\sum_{j \geqslant N(\delta)} \frac{1}{\mu_{j}} \leqslant \delta
$$

for all $\delta>0$.

We have the following result: 
Proposition 3.2. - Given $\xi>0$, a function $N$ as above and $T>0$, there exists a positive constant $C$, which depends on $\xi, N$ and $T$, such that

$$
\int_{0}^{T}\left|\sum_{k=1}^{+\infty} a_{k} \mathrm{e}^{-\mu_{k} t}\right|^{2} \mathrm{~d} t \geqslant \frac{C}{\left(\sum_{i=1}^{+\infty} \frac{1}{\mu_{i}}\right)} \sum_{k=1}^{+\infty} \frac{\left|a_{k}\right|^{2}}{\mu_{k}} \mathrm{e}^{-2 \mu_{k} T},
$$

for all $\left\{\mu_{j}\right\}_{j} \geqslant 1 \in \mathcal{L}(\xi, N)$ and all sequence $\left\{a_{j}\right\}_{j} \geqslant 1$ of real numbers.

Before proving this proposition we complete the proof of (50). We introduce the sequences $\left\{\bar{\lambda}_{k}^{\varepsilon}\right\}_{k \geqslant 1}$ given by

$$
\bar{\lambda}_{k}^{\varepsilon}= \begin{cases}\lambda_{k}^{\varepsilon} & \text { if } k \leqslant D \varepsilon^{-1}, \\ k^{2} \pi^{2} / \rho_{m} & \text { if } k>D \varepsilon^{-1} .\end{cases}
$$

Taking (18) and (22) into account it is easy to prove that there exist $\xi>0$ and a function $N$ as above such that conditions (51)-(53) are satisfied uniformly by the sequences $\left\{\bar{\lambda}_{k}^{\varepsilon}\right\}_{k \geqslant 1}$ for all $\varepsilon \in(0,1)$. Applying Proposition 3.2 to the sequence $\left\{\bar{\lambda}_{k}^{\varepsilon}\right\}_{k} \geqslant 1$ we obtain (50).

Proof of Proposition 3.2. - Let

$$
\Lambda=\left\{\mu_{j}\right\}_{j} \geqslant 1
$$

be a sequence of positive real numbers and let $E(\Lambda, T)$ denote the smallest closed subspace of $L^{2}(0, T)$ containing the functions $\mathrm{e}^{-\mu_{j} t}, j=1,2, \ldots$ It is well-known, see for instance [20], that $E(\Lambda, T)$ is a proper subspace of $L^{2}(0, T)$ if and only if

$$
\sum_{j=1}^{\infty} \frac{1}{\mu_{j}}<+\infty
$$

The following result can be found in [9], Theorem 1.3, p. 47:

Lemma 3.1. - Let $0<T<\infty$ and $\Lambda \in \mathcal{L}(\xi, N)$. Then the restriction mapping

$$
S: E(\Lambda, \infty) \rightarrow E(\Lambda, T),
$$

has a bounded inverse, i.e.

$$
\left\|S^{-1}\right\| \leqslant C,
$$

where $C$ is a positive constant determined uniquely by $\xi, N$ and $T$.

In other words, for every $\Lambda \in \mathcal{L}(\xi, N)$ there exists a positive constant $C(\xi, N, T)$ such that

$$
\int_{0}^{T}\left|\sum_{j=1}^{+\infty} a_{j} \mathrm{e}^{-\mu_{j} t}\right|^{2} \mathrm{~d} t \geqslant C(\zeta, N, T) \int_{0}^{+\infty}\left|\sum_{j=1}^{+\infty} a_{j} \mathrm{e}^{-\mu_{j} t}\right|^{2} \mathrm{~d} t
$$


for all $\sum_{j=1}^{+\infty} a_{j} \mathrm{e}^{-\mu_{j} t} \in E(\Lambda, T)$.

Let $\left\{q_{k}\right\}_{k \geqslant 1}$ be a biorthogonal sequence in $L^{2}(0, \infty)$ for $\left\{\mathrm{e}^{-\mu_{j} t}\right\}_{j} \geqslant 1$, i.e.

$$
\int_{0}^{\infty} q_{k} \mathrm{e}^{-\mu_{j} t}= \begin{cases}1 & \text { if } k=j \\ 0 & \text { if } k \neq j\end{cases}
$$

By (60),

$$
\left|\int_{0}^{\infty}\left(\sum_{k=1}^{+\infty} a_{k} \mathrm{e}^{-\mu_{k} t}\right) q_{i} \mathrm{~d} t\right|^{2}=\left|a_{i}\right|^{2}
$$

We have that

$$
\left|\int_{0}^{\infty}\left(\sum_{k=1}^{+\infty} a_{k} \mathrm{e}^{-\mu_{k} t}\right) q_{i} \mathrm{~d} t\right|^{2} \leqslant\left\|q_{i}\right\|_{L^{2}(0, \infty)}^{2} \int_{0}^{\infty}\left|\sum_{k=1}^{+\infty} a_{k} \mathrm{e}^{-\mu_{k} t}\right|^{2} \mathrm{~d} t .
$$

By (61) and (62) we get

$$
\int_{0}^{\infty}\left|\sum_{k=1}^{+\infty} a_{k} \mathrm{e}^{-\mu_{k} t}\right|^{2} \mathrm{~d} t \geqslant \frac{\left|a_{i}\right|^{2}}{\left\|q_{i}\right\|_{L^{2}(0, \infty)}^{2}}, \quad \text { for all } i=1, \ldots,+\infty .
$$

Multiplying (63) by $\frac{1}{\mu_{i}}$ we get

$$
\sum_{i=1}^{+\infty} \frac{1}{\mu_{i}} \int_{0}^{\infty}\left|\sum_{k=1}^{+\infty} a_{k} \mathrm{e}^{-\mu_{k} t}\right|^{2} \mathrm{~d} t \geqslant \sum_{i=1}^{+\infty} \frac{\left|a_{i}\right|^{2}}{\mu_{i}\left\|q_{i}\right\|_{L^{2}(0, \infty)}^{2}}
$$

or equivalently

$$
\int_{0}^{+\infty}\left|\sum_{k=1}^{+\infty} a_{k} \mathrm{e}^{-\mu_{k} t}\right|^{2} \mathrm{~d} t \geqslant \frac{1}{\left(\sum_{i=1}^{+\infty} \frac{1}{\mu_{i}}\right)} \sum_{k=1}^{+\infty} \frac{\left|a_{k}\right|^{2}}{\mu_{k}\left\|q_{k}\right\|_{L^{2}(0,+\infty)}^{2}} .
$$

The following result can be found in [9], Theorem 1.1, p. 46:

LEMMA 3.2. - Given $\xi>0$ and a decreasing function

$$
N:(0,+\infty) \rightarrow \mathbb{N}
$$

there exists a function $K:(0, \infty) \rightarrow(0, \infty)$, depending uniquely on $\xi$ and $N$, such that

$$
\left\|q_{k}\right\|_{L^{2}(0, \infty)}^{2} \leqslant K(\delta) \mathrm{e}^{\delta \mu_{k}},
$$

for all $k=1,2, \ldots,+\infty, \delta>0$ and $\left\{\mu_{k}\right\}_{k \geqslant 1} \in \mathcal{L}(\zeta, N)$, where $\left\{q_{k}\right\}_{k \geqslant 1}$ is a biorthogonal sequence for the sequence $\left\{\mathrm{e}^{-\mu_{k} t}\right\}_{k} \geqslant 1$ in $L^{2}(0, \infty)$. 
Applying Lemma 3.2,

$$
\int_{0}^{+\infty}\left|\sum_{k=1}^{+\infty} a_{k} \mathrm{e}^{-\mu_{k} t}\right|^{2} \mathrm{~d} t \geqslant \frac{1}{\left(\sum_{i=1}^{+\infty} \frac{1}{\mu_{i}}\right)} \sum_{k=1}^{+\infty} \frac{\left|a_{k}\right|^{2}}{\mu_{k}\left\|q_{k}\right\|_{L^{2}(0, \infty)}^{2}} \geqslant \frac{1}{\left(\sum_{i=1}^{+\infty} \frac{1}{\mu_{i}}\right)} \sum_{k=1}^{+\infty} \frac{\left|a_{k}\right|^{2}}{\mu_{k} K(\delta)} \mathrm{e}^{-\delta \mu_{k}} .
$$

Taking $\delta=2 T$,

$$
\int_{0}^{+\infty}\left|\sum_{k=1}^{+\infty} a_{k} \mathrm{e}^{-\mu_{k} t}\right|^{2} \mathrm{~d} t \geqslant \frac{1}{K(2 T)\left(\sum_{i=1}^{+\infty} \frac{1}{\mu_{i}}\right)} \sum_{k=1}^{+\infty} \frac{\left|a_{k}\right|^{2}}{\mu_{k}} \mathrm{e}^{-2 \mu_{k} T} .
$$

This concludes the proof of Proposition 3.2.

\section{Global non-uniform controllability}

This section is devoted to prove inequality (38).

We have the following result:

PROPOSITION 4.1. - Assume that $\rho \in W^{2, \infty}(\mathbb{R})$ is a periodic function satisfying (1). Let $T>0$. Then, for any $u_{0} \in L^{2}(0,1)$ there exists a control $f^{\varepsilon} \in L^{2}(0, T)$ such that the solution of (3) satisfies

$$
u^{\varepsilon}(T)=0 .
$$

Moreover, there exist positive constants $C_{1}$ and $C_{2}$, independent of $\varepsilon \in(0,1)$, such that

$$
\left\|f^{\varepsilon}\right\|_{L^{2}(0, T)} \leqslant C_{1} \mathrm{e}^{C_{2} / \varepsilon^{4 / 3}}\left\|u_{0}\right\|_{L^{2}(0,1)},
$$

for all $u_{0} \in L^{2}(0,1)$.

Remark 4.1. - The fact that Eq. (3) is null-controllable when $\rho$ belongs to $W^{2, \infty}(\mathbb{R})$ is not new. The new contribution of Proposition 4.1 is estimate (70) which provides a first rough estimate on how does the control depend on $\varepsilon$ as $\varepsilon \rightarrow 0$. Note that, according to Theorem 1.1, estimate (70) is far from being sharp since one can actually prove that the controls remain bounded as $\varepsilon \rightarrow 0$. But, as an intermediate step in the proof of Theorem 1.1, we need to first prove this weaker version.

Proof. - Arguing as in the begining of the proof of Proposition 3.1, the proof of Proposition 4.1 can be easily reduced to prove the following observability inequality:

Proposition 4.2. - For any $T>0$ there exist positive constants $C_{1}$ and $C_{2}$, independent of $0<\varepsilon<1$, such that

$$
\left\|\phi^{\varepsilon}(x, 0)\right\|_{L^{2}(0,1)}^{2} \leqslant C_{1} \mathrm{e}^{C_{2} / \varepsilon^{4 / 3}} \int_{0}^{T}\left|\phi_{x}^{\varepsilon}(1, t)\right|^{2} \mathrm{~d} t,
$$

for every $\phi^{\varepsilon}$ solution of $(11)$ and for all $\varepsilon \in(0,1)$. 
Proof of Proposition 4.2. - Let us consider the variable coefficient adjoint heat equation

$$
\begin{cases}a(x) \phi_{t}+\phi_{x x}=0, & 0<x<1,0<t<T, \\ \phi(0, t)=\phi(1, t)=0, & 0<t<T, \\ \phi(x, T)=\phi^{0}(x), & 0<x<1,\end{cases}
$$

with $a \in W^{2, \infty}(\mathbb{R})$ such that

$$
0<a_{m} \leqslant a(x) \leqslant a_{M}<+\infty,
$$

for all $x \in(0,1)$. The following holds:

Lemma 4.1. - For any time $T>0$ there exist positive constants $C_{1}(T), C_{2}$ and $C_{3}(T)$ such that

$$
\|\phi(x, 0)\|_{L^{2}(0,1)}^{2} \leqslant C_{1}(T) \mathrm{e}^{\left(C_{2}\|a\|_{W^{1, \infty}(I R)}^{4 / 3}+C_{3}(T)\|a\|_{W^{2}, \infty}^{2 / 3}\right)} \int_{0}^{T}\left|\phi_{x}(1, t)\right|^{2} \mathrm{~d} t
$$

for every solution $\phi$ of (72) and for every $a \in W^{2, \infty}(\mathbb{R})$ verifying (73).

Applying Lemma 4.1 to the solutions of (72) with $a(x)=\rho\left(\frac{x}{\varepsilon}\right)$ we obtain (71).

This completes the proof of Propositions 4.2 and 4.1. It only remains to prove Lemma 4.1.

The proof of Lemma 4.1 can be done reproducing carefully the developments in [10] and [11] on Global Carleman Inequalities. In order to obtain (74) one has first to reduce the problem to the heat operator with a lower order potential an to prove the Carleman inequalities paying special attention to how the various constants entering in the inequalities depend on the potential.

In order to make the article easier to read this proof is given in an appendix at the end of this paper.

\section{Proof of the main controllability result}

This section is devoted to complete the proof of the uniform controllability result of Theorem 1.1. Note that the proof of the convergence of the control stated in Theorem 1.1 is left to Section 6.

We follow the control strategy presented in Section 2.

We fix a control time $T>0$ and we divide it into three subintervals $I_{1}=\left[0, \frac{T}{3}\right], I_{2}=$ $\left[\frac{T}{3}, \frac{2 T}{3}\right]$ and $I_{3}=\left[\frac{2 T}{3}, T\right]$.

By the first part of Theorem 3.1, for any $u_{0} \in L^{2}(0,1)$ there exists a control $f_{\frac{T}{6}}^{\varepsilon} \in$ $L^{2}\left(0, \frac{T}{6}\right)$ such that the solution of (3) verifies that

$$
\Pi_{k \leqslant\left[D \varepsilon^{-1}\right]} u^{\varepsilon}\left(\frac{T}{6}\right)=0 .
$$


Moreover, according to Theorem 3.1, we deduce the existence of a positive constant $C$ which depends on $T$ but is independent of $\varepsilon \in(0,1)$ such that

$$
\left\|f_{\frac{T}{6}}^{\varepsilon}\right\|_{L^{2}\left(0, \frac{T}{6}\right)} \leqslant C\left\|u_{0}\right\|_{L^{2}(0,1)}
$$

for all $u_{0} \in L^{2}(0,1)$ and $\varepsilon \in(0,1)$.

Let us consider in the first time interval $I_{1}$ the control

$$
f_{1}^{\varepsilon}= \begin{cases}f_{\frac{T}{6}}^{\varepsilon} & 0 \leqslant t \leqslant \frac{T}{6} \\ 0 & \frac{T}{6}<t \leqslant \frac{T}{3}\end{cases}
$$

We deduce that the solution of (26) verifies (27). On the other hand, we have, by classical energy estimates,

$$
\left\|u^{\varepsilon}\left(\frac{T}{3}\right)\right\|_{L^{2}(0,1)} \leqslant C(T)\left(\left\|u_{0}\right\|_{L^{2}(0,1)}+\left\|f_{1}^{\varepsilon}\right\|_{L^{2}\left(0, \frac{T}{3}\right)}\right) .
$$

At this point it is important to observe that (76) holds because $f_{1}^{\varepsilon}$ vanish on $\left[\frac{T}{6}, \frac{T}{3}\right]$. In fact it would suffice $f_{1}^{\varepsilon}$ to vanish on any interval $\left[\frac{T}{3}-\delta, \frac{T}{3}\right]$, for any $\delta>0$.

In order to prove (76) we proceed as follows: Without lost of generality we may assume that $u^{0}=0$ since in the case where $f_{1}^{\varepsilon}=0$, (76) holds obviously. Multiplying

$$
\begin{cases}\rho\left(\frac{x}{\varepsilon}\right) u_{t}^{\varepsilon}-u_{x x}^{\varepsilon}=0, & 0<x<1,0<t<\frac{T}{6}, \\ u^{\varepsilon}(0, t)=0, \quad u^{\varepsilon}(1, t)=f_{1}^{\varepsilon}(t), & 0<t<\frac{T}{6}, \\ u^{\varepsilon}(x, 0)=0, & 0<x<1\end{cases}
$$

by $\phi^{\varepsilon}$ solution of the adjoint system

$$
\begin{cases}\rho\left(\frac{x}{\varepsilon}\right) \phi_{t}^{\varepsilon}+\phi_{x x}^{\varepsilon}=0, & 0<x<1,0<t<\frac{T}{6}, \\ \phi^{\varepsilon}(0, t)=\phi^{\varepsilon}(1, t)=0, & 0<t<\frac{T}{6} \\ \phi^{\varepsilon}\left(x, \frac{T}{6}\right)=\phi^{0}(x), & 0<x<1\end{cases}
$$

and integrating by parts we have that

$$
\int_{0}^{\frac{T}{6}} \int_{0}^{1} \rho\left(\frac{x}{\varepsilon}\right) u^{\varepsilon}\left(\frac{T}{6}\right) \phi^{0}(x) \mathrm{d} x \mathrm{~d} t=-\int_{0}^{\frac{T}{6}} f_{1}^{\varepsilon} \phi_{x}^{\varepsilon}(1, t) .
$$

We have the following lemma:

LEMMA 5.1. - Let us consider the following system:

$$
\begin{cases}\rho(x / \varepsilon) \phi_{t}^{\varepsilon}+\phi_{x x}^{\varepsilon}=0, & 0<x<1,0<t<T, \\ \phi^{\varepsilon}(0, t)=\phi^{\varepsilon}(1, t)=0, & 0<t<T \\ \phi^{\varepsilon}(x, T)=\phi^{0}(x), & 0<x<1\end{cases}
$$

with $\phi^{0} \in H^{2} \cap H_{0}^{1}(0,1)$. Then, 


$$
\begin{aligned}
\phi^{\varepsilon} & \rightarrow \phi \text { weakly in } L^{2}\left(0, T ; H^{2} \cap H_{0}^{1}(0,1)\right), \quad \text { as } \varepsilon \rightarrow 0, \\
\phi^{\varepsilon} & \rightarrow \phi \text { strongly in } C\left([0, T] ; H^{s}\right) \text { for all } s<2, \quad \text { as } \varepsilon \rightarrow 0, \\
\phi_{x}^{\varepsilon}(1, t) & \rightarrow \phi_{x}(1, t) \text { strongly in } C([0, T]), \quad \text { as } \varepsilon \rightarrow 0,
\end{aligned}
$$

where $\phi$ is the solution of the limit system

$$
\begin{cases}\bar{\rho} \phi_{t}+\phi_{x x}=0, & 0<x<1,0<t<T, \\ \phi(0, t)=\phi(1, t)=0, & 0<t<T, \\ \phi(x, T)=\phi^{0}(x), & 0<x<1 .\end{cases}
$$

Proof. - The function $\psi^{\varepsilon}=\phi_{t}^{\varepsilon}$ verifies

$$
\begin{cases}\rho(x / \varepsilon) \psi_{t}^{\varepsilon}+\psi_{x x}^{\varepsilon}=0, & 0<x<1,0<t<T, \\ \psi^{\varepsilon}(0, t)=\psi^{\varepsilon}(1, t)=0, & 0<t<T \\ \psi^{\varepsilon}(x, T)=-\phi_{x x}^{0} / \rho(x / \varepsilon), & 0<x<1\end{cases}
$$

where $\psi^{\varepsilon}(x, T)=-\phi_{x x}^{0} / \rho(x / \varepsilon)$ is bounded in $L^{2}(0,1)$. Then,

$$
\phi_{t}^{\varepsilon} \text { is bounded in } C\left([0, T] ; L^{2}(0,1)\right) \text {, }
$$

and consequently,

$$
\left\{\begin{array}{l}
\phi^{\varepsilon} \text { is bounded in } C\left([0, T] ; H^{2} \cap H_{0}^{1}(0,1)\right), \\
\phi^{\varepsilon} \text { is bounded in } C^{1}\left([0, T] ; L^{2}(0,1)\right) .
\end{array}\right.
$$

By the Aubin-Lions theorem we deduce that $\phi^{\varepsilon}$ is relatively compact in $C\left([0, T] ; H^{s}(0,1)\right)$, for all $0<s<2$. By extracting subsequences we have that

$$
\begin{aligned}
\phi^{\varepsilon} & \rightarrow \phi \text { weakly in } L^{2}\left(0, T ; H^{2} \cap H_{0}^{1}(0,1)\right), \quad \text { as } \varepsilon \rightarrow 0, \\
\phi^{\varepsilon} & \rightarrow \phi \text { strongly in } C\left([0, T] ; H^{s}\right) \text { for all } s<2, \quad \text { as } \varepsilon \rightarrow 0, \\
\phi_{x}^{\varepsilon}(1, t) & \rightarrow \phi_{x}(1, t) \text { strongly in } C([0, T]), \quad \text { as } \varepsilon \rightarrow 0 .
\end{aligned}
$$

Passing to the limit in the variational formulation of (80) it is easy to see that the limit $\phi$ is the solution of system (82).

Let us go back to the proof of (76). In the right hand side of (79) we have that, by Lemma 5.1,

$$
\left|\int_{0}^{\frac{T}{6}} f_{1}^{\varepsilon} \phi_{x}^{\varepsilon}(1, t) \mathrm{d} t\right| \leqslant\left\|f_{1}^{\varepsilon}\right\|_{L^{2}\left(0, \frac{T}{6}\right)}\left\|\phi_{x}^{\varepsilon}(1, t)\right\|_{L^{2}\left(0, \frac{T}{6}\right)} \leqslant C(T)\left\|f_{1}^{\varepsilon}\right\|_{L^{2}\left(0, \frac{T}{6}\right)}\left\|\phi^{\varepsilon}\right\|_{H^{2} \cap H_{0}^{1}(0,1)} .
$$

Thus, by duality,

$$
\left\|u^{\varepsilon}(T / 6)\right\|_{H^{-2}(0,1)} \leqslant C(T)\left\|f_{1}^{\varepsilon}\right\|_{L^{2}\left(0, \frac{T}{6}\right)} .
$$

Remark 5.1. - The arguments we have used in the proof of Lemma 5.1 allow to show that, when $\phi_{x} \in H^{s}(0,1) \cap H_{0}^{1}(0,1)$, with $s>3 / 2$, then

$$
\left\{\phi_{x}^{\varepsilon}(1, t)\right\} \rightarrow \phi_{x}(1, t) \text { stongly in } C([0, T]) .
$$


This is because, under this assumption on $\phi^{0}$, it can be shown that $\phi^{\varepsilon}$ is relatively compact in $C\left([0, T] ; H^{s^{\prime}}(0,1)\right)$ for all $0<s^{\prime}<s$.

In what concerns (87), this allows to get a uniform bound on $u^{\varepsilon}(T / 6)$ in all the Sobolev spaces $H^{-s}(0,1)$, for all $s>3 / 2$.

Now, taking the regularizing effect of the solutions of (77) into account, we have that

$$
\begin{aligned}
\int_{0}^{1} \rho\left(\frac{x}{\varepsilon}\right)\left|u^{\varepsilon}(T / 3)\right|^{2} \mathrm{~d} x & =\sum_{k \geqslant 1}\left|a_{k}\right|^{2} \mathrm{e}^{-\frac{2 \lambda_{k}^{\varepsilon} T}{3}}=\sum_{k \geqslant 1} \frac{\left|a_{k}\right|^{2}}{\left(\lambda_{k}^{\varepsilon}\right)^{2}} \mathrm{e}^{-\frac{2 \lambda_{k}^{\varepsilon} T}{6}}\left(\lambda_{k}^{\varepsilon}\right)^{2} \mathrm{e}^{-\frac{2 \lambda_{k}^{\varepsilon} T}{6}} \\
& \leqslant C(T) \sum_{k \geqslant 1} \frac{\left|a_{k}\right|^{2}}{\left(\lambda_{k}^{\varepsilon}\right)^{2}} \mathrm{e}^{-\frac{2 \lambda_{k}^{\varepsilon} T}{6}} \leqslant C(T)\left\|u^{\varepsilon}(T / 6)\right\|_{H^{-2}(0,1)},
\end{aligned}
$$

consequently,

$$
\left\|u^{\varepsilon}(T / 3)\right\|_{L^{2}(0,1)} \leqslant C(T)\left\|u^{\varepsilon}(T / 6)\right\|_{H^{-2}(0,1)},
$$

with $C(T)>0$ independent of $\varepsilon \in(0,1)$.

Therefore, by (87) and (88),

$$
\|u(T / 3)\|_{L^{2}(0,1)} \leqslant C(T)\left\|f_{1}^{\varepsilon}\right\|_{L^{2}\left(0, \frac{T}{3}\right)},
$$

where $C(T)$ is a positive constant independent of $\varepsilon \in(0,1)$.

This completes the proof of (76).

In the second time interval $I_{2}=\left[\frac{T}{3}, \frac{2 T}{3}\right]$ we let the system to evolve freely. In other words, we solve the uncontrolled system (31) with initial datum $v_{0}^{\varepsilon}=u\left(\frac{T}{3}\right), u$ being the solution of (8) obtained in the first time interval $I_{1}$.

We have the following result:

LEMMA 5.2. - Let $u^{\varepsilon}$ be the solution of (3) with $f=0$ and with an initial $u_{0} \in$ $L^{2}(0,1)$ such that

$$
\Pi_{k \leqslant\left[D \varepsilon^{-1}\right]} u_{0}=0
$$

Then

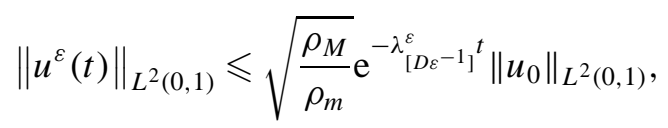

for all $t \geqslant 0$.

Proof. - We decompose the initial datum in Fourier series:

$$
u_{0}=\sum_{j \geqslant D \varepsilon^{-1}} a_{j} \varphi_{k}^{\varepsilon}(x)
$$

The solution of (3) with $f=0$ may be written as:

$$
u^{\varepsilon}(t)=\sum_{j \geqslant\left[D \varepsilon^{-1}\right]} a_{j} \mathrm{e}^{-\lambda_{k}^{\varepsilon} t} \varphi_{k}^{\varepsilon}(x) .
$$


Then, due to the orthogonality of the eigenfunctions $\varphi_{k}^{\varepsilon}(x)$,

$$
\begin{aligned}
\left\|u^{\varepsilon}(t)\right\|_{L^{2}(0,1)}^{2} & \leqslant \frac{1}{\rho_{m}} \int_{0}^{1} \rho\left(\frac{x}{\varepsilon}\right)\left|u^{\varepsilon}(x, t)\right|^{2} \mathrm{~d} x=\frac{1}{\rho_{m}} \sum_{j \geqslant\left[D \varepsilon^{-1}\right]}\left|a_{j}\right|^{2} \mathrm{e}^{-2 \lambda_{k}^{\varepsilon} t} \\
& \leqslant \frac{\mathrm{e}^{-2 \lambda_{\left[D \varepsilon^{-1}\right]^{t}}^{t}}}{\rho_{m}} \sum_{j \geqslant\left[D \varepsilon^{-1}\right]}\left|a_{j}\right|^{2}=\frac{\mathrm{e}^{-2 \lambda_{\left[D \varepsilon^{-1}\right]^{t}}^{t}}}{\rho_{m}} \int_{0}^{1} \rho\left(\frac{x}{\varepsilon}\right)\left|u_{0}(x)\right|^{2} \mathrm{~d} x \\
& \leqslant \frac{\rho_{M}}{\rho_{m}} \mathrm{e}^{-2 \lambda_{\left[D \varepsilon^{-1}\right]^{t}}^{t}} \int_{0}^{1}\left|u_{0}(x)\right|^{2} \mathrm{~d} x=\frac{\rho_{M}}{\rho_{m}} \mathrm{e}^{-2 \lambda_{\left[D \varepsilon^{-1}\right]^{t}}^{t}\left\|u_{0}\right\|_{L^{2}(0,1)}^{2} .}
\end{aligned}
$$

According to (91), the solution of system (31) verifies

$$
\left\|u^{\varepsilon}(t)\right\|_{L^{2}(0,1)} \leqslant \sqrt{\frac{\rho_{M}}{\rho_{m}}} \mathrm{e}^{-\lambda_{\left[D \varepsilon^{-1}\right]}^{\varepsilon}\left(t-\frac{T}{3}\right)}\left\|u^{\varepsilon}\left(\frac{T}{3}\right)\right\|_{L^{2}(0,1)},
$$

for all $\frac{T}{3} \leqslant t \leqslant \frac{2 T}{3}$. In particular

$$
\left\|u^{\varepsilon}\left(\frac{2 T}{3}\right)\right\|_{L^{2}(0,1)} \leqslant \sqrt{\frac{\rho_{M}}{\rho_{m}}} \mathrm{e}^{-\lambda_{\left[D \varepsilon^{-1}\right]}^{\varepsilon} T / 3}\left\|u^{\varepsilon}\left(\frac{T}{3}\right)\right\|_{L^{2}(0,1)} .
$$

Taking (89), (96) and (18) into account we conclude that

$$
\left\|u^{\varepsilon}\left(\frac{2 T}{3}\right)\right\|_{L^{2}(0,1)} \leqslant C(T) \mathrm{e}^{-\pi^{2} D^{2} T /\left(3 \rho_{M} \varepsilon^{2}\right)}\left\|u^{\varepsilon}\left(\frac{T}{3}\right)\right\|_{L^{2}(0,1)},
$$

where $C(T)$ is a positive constant independent of $\varepsilon \in(0,1)$.

In the last time interval $I_{3}$ we control to zero the datum $w_{0}^{\varepsilon}=u^{\varepsilon}\left(\frac{2 T}{3}\right)$ obtained in the two previous steps. In other words, we solve (36) with the control $f_{2}^{\varepsilon}$ given by Theorem 4.1. Then we have

$$
u^{\varepsilon}(T)=0,
$$

as we wanted to prove. On the other hand,

$$
\begin{aligned}
\left\|f_{2}^{\varepsilon}\right\|_{L^{2}\left(\frac{2 T}{3}, T\right)} & \leqslant C(T) \mathrm{e}^{C(T) / \varepsilon^{4 / 3}}\left\|w_{0}^{\varepsilon}\right\|_{L^{2}(0,1)} \\
& \leqslant C(T) \mathrm{e}^{C(T) / \varepsilon^{4 / 3}} \mathrm{e}^{-\pi^{2} D^{2} T /\left(3 \rho_{M} \varepsilon^{2}\right)}\left\|u^{\varepsilon}\left(\frac{T}{3}\right)\right\|_{L^{2}(0,1)} \\
& \leqslant C(T) \mathrm{e}^{C(T) / \varepsilon^{4 / 3}} \mathrm{e}^{-\pi^{2} D^{2} T /\left(3 \rho_{M} \varepsilon^{2}\right)}\left\|u_{0}\right\|_{L^{2}(0,1)} .
\end{aligned}
$$

In particular

$$
\left\|f_{2}^{\varepsilon}\right\|_{L^{2}\left(\frac{2 T}{3}, T\right)} \leqslant C_{1} \mathrm{e}^{-C_{2} / \varepsilon^{2}} \quad \text { as } \varepsilon \rightarrow 0,
$$

for suitable $C_{1}, C_{2}>0$. 
This argument shows that the control

$$
f^{\varepsilon}= \begin{cases}f_{1}^{\varepsilon} & 0 \leqslant t \leqslant T / 3 \\ 0 & T / 3<t<2 T / 3 \\ f_{2}^{\varepsilon} & 2 T / 3 \leqslant t \leqslant T\end{cases}
$$

is such that the null controllability condition (98) holds and, according to (75) and (99),

$$
\left\|f^{\varepsilon}\right\|_{L^{2}(0, T)} \leqslant C\left\|u_{0}\right\|_{L^{2}(0,1)},
$$

where $C$ is a positive constant independent of $0<\varepsilon<1$.

This completes the proof of the uniform null controllabilitty result of Theorem 1.1.

\section{The limit process}

This section is devoted to prove the convergence of the controls stated in Theorem 1.1.

We fix $T>0$ and $u_{0} \in L^{2}(0,1)$. We then consider the control $f^{\varepsilon} \in L^{2}(0, T)$ such that the solution of (3) verifies (4). Note that, by Theorem 1.1,

$$
\left\|f^{\varepsilon}\right\|_{L^{2}(0, T)} \leqslant C\left\|u_{0}\right\|_{L^{2}(0,1)},
$$

with $C>0$ independent of $\varepsilon \in(0,1)$.

By duality, it can be proved that the control $f^{\varepsilon}$ may be chosen to be of the form

$$
f^{\varepsilon}(t)=\varphi_{x}^{\varepsilon}(1, t),
$$

for all $t \in(0, T)$, where $\varphi^{\varepsilon}$ is the solution of

$$
\begin{cases}\rho\left(\frac{x}{\varepsilon}\right) \phi_{t}^{\varepsilon}+\phi_{x x}^{\varepsilon}=0, & 0<x<1,0<t<T, \\ \phi^{\varepsilon}(0, t)=\phi^{\varepsilon}(1, t)=0, & 0<t<T, \\ \phi^{\varepsilon}(x, T)=\phi^{0}(x), & 0<x<1,\end{cases}
$$

which minimizes the functional

$$
J^{\varepsilon}\left(\phi^{\varepsilon}\right)=\frac{1}{2} \int_{0}^{T}\left|\phi_{x}^{\varepsilon}(1, t)\right|^{2} \mathrm{~d} t-\int_{0}^{1} \rho\left(\frac{x}{\varepsilon}\right) u_{0}(x) \phi^{\varepsilon}(x, 0) \mathrm{d} x,
$$

in the space

$$
F^{\varepsilon}=\left\{\begin{array}{cl}
g \in L^{2}(0, T): & g=\phi_{x}^{\varepsilon}(1, t) \text { for some solution } \phi \text { of }(104) \\
& \text { with } \phi^{\varepsilon} \in L_{\text {loc }}^{2}\left(0, T ; H^{2} \cap H_{0}^{1}(0,1)\right)
\end{array}\right\},
$$

endowed with the norm

$$
\|g\|_{F^{\varepsilon}}=\left(\int_{0}^{T}\left|\phi_{x}^{\varepsilon}(1, t)\right|^{2} \mathrm{~d} t\right)^{1 / 2}
$$


Note that when we choose the control $f^{\varepsilon}(t)=\varphi_{x}^{\varepsilon}(1, t)$ the bound (102) is kept, i.e. the sequence of minimizers of the funtionals $J^{\varepsilon}$ are such that

$$
\int_{0}^{T}\left|\varphi_{x}^{\varepsilon}(1, t)\right|^{2} \mathrm{~d} t \leqslant C,
$$

where $C$ is a positive constant independent of $\varepsilon \in(0,1)$. This is so since, actually, the control $f^{\varepsilon}(t)=\varphi_{x}^{\varepsilon}(1, t)$ one obtains minimizing $J^{\varepsilon}$ on $F^{\varepsilon}$ is the one of minimal $L^{2}(0, T)$-norm.

By extracting subsequences (that we still denote by the index $\varepsilon$ ) we deduce that

$$
\varphi_{x}^{\varepsilon}(1, t) \rightarrow \varsigma(t) \text { weakly in } L^{2}(0, T), \quad \text { as } \varepsilon \rightarrow 0 .
$$

First of all we are going to prove that the function $\varsigma(t)$ is a control of the limit system (6).

The Euler equation satisfied by the sequence of minimizers $\left\{\varphi_{x}^{\varepsilon}\right\}$ of the functionals $F^{\varepsilon}$ may be written as

$$
\left\{\begin{array}{l}
\int_{0}^{T} \varphi_{x}^{\varepsilon}(1, t) \phi_{x}^{\varepsilon}(1, t) \mathrm{d} t-\int_{0}^{1} \rho\left(\frac{x}{\varepsilon}\right) u_{0}(x) \phi^{\varepsilon}(x, 0) \mathrm{d} x=0, \\
\text { for all } \phi^{\varepsilon} \text { solution of }(104) .
\end{array}\right.
$$

Let us fix $\phi^{0} \in H^{2} \cap H_{0}^{1}(0,1)$ as the initial datum of (104). By Lemma 5.1,

$$
\phi_{x}^{\varepsilon}(1, t) \rightarrow \phi_{x}(1, t) \text { strongly in } L^{2}(0, T),
$$

and

$$
\phi^{\varepsilon}(x, 0) \rightarrow \phi(x, 0) \text { strongly in } L^{2}(0,1),
$$

where $\phi$ is the solution of the adjoint limit system

$$
\begin{cases}\bar{\rho} \phi_{t}+\phi_{x x}=0, & 0<x<1,0<t<T, \\ \phi(0, t)=\phi(1, t)=0, & 0<t<T, \\ \phi(x, T)=\phi^{0}(x), & 0<x<1 .\end{cases}
$$

Using (111) and (112) and passing to the limit $\varepsilon \rightarrow 0$ in (110) we obtain that

$$
\int_{0}^{T} \varsigma(t) \phi_{x}(1, t) \mathrm{d} t-\int_{0}^{1} \bar{\rho} u_{0}(x) \phi(x, 0) \mathrm{d} x=0,
$$

for all $\phi$ solution of (113) with $\phi^{0} \in H^{2} \cap H_{0}^{1}(0,1)$.

But then the solution $u$ of the controlled heat equation (6) with control $f=\varsigma$ is such that

$$
u(x, T)=0, \quad \text { for all } x \in(0,1)
$$


Indeed, multiplying in (6) by $\phi$ solution of (113) and integrating by parts we deduce that

$$
\int_{0}^{T} \varsigma(t) \phi_{x}(1, t) \mathrm{d} t+\left.\int_{0}^{1} \bar{\rho} u(x, t) \phi(x, t) \mathrm{d} x\right|_{0} ^{T}=0,
$$

which combined with (114) implies that

$$
\int_{0}^{1} u(x, T) \phi^{0} \mathrm{~d} x=0, \quad \text { for all } \phi^{0} \in H^{2} \cap H_{0}^{1}(0,1),
$$

and this implies (115).

Consequently the weak limit $\zeta(t)$ is a null control for the limit equation (6).

We are now going to prove the following two facts to complete the proof of Theorem 1.1:

(a) The limit $\varsigma$ is uniquely determined, i.e. it is independent of the subsequence;

(b) The convergence (109) holds in the strong topology of $L^{2}(0, T)$.

We proceed as follows. According to the first statement of Theorem 1.1 and by Theorem 1.2, the uniform observability

$$
\left\|\phi^{\varepsilon}(\cdot, 0)\right\|_{L^{2}(0,1)}^{2} \leqslant C(T) \int_{0}^{T}\left|\phi_{x}^{\varepsilon}(1, t)\right|^{2} \mathrm{~d} t,
$$

holds for every $\phi^{\varepsilon}$ solution of system (11) and for any $T>0$. Consequently (118) holds also in any interval of the form $(\tau, T)$, with $0<\tau<T$ and provides an estimate of $\phi^{\varepsilon}$ at $t=\tau$. Applying this estimate to $\varphi^{\varepsilon}$, the minimizer of the functional $J^{\varepsilon}$ in $F^{\varepsilon}$, we deduce that

$$
\left\|\varphi^{\varepsilon}(\cdot, \tau)\right\|_{L^{2}(0,1)}^{2} \leqslant C(T-\tau) \int_{\tau}^{T}\left|\varphi_{x}^{\varepsilon}(1, t)\right|^{2} \mathrm{~d} t,
$$

with $0 \leqslant \tau<T$.

Taking (108) and (119) into account we deduce that

$$
\left\|\varphi^{\varepsilon}(\cdot, \tau)\right\|_{L^{2}(0,1)}^{2} \leqslant C(T-\tau), \quad \text { for all } 0 \leqslant \tau<T,
$$

with another positive constant $C(T-\tau)$ independent of $\varepsilon \in(0,1)$.

By the regularizing effect of Eq. (11) the bound (120) implies that

$$
\left\|\varphi^{\varepsilon}(\cdot, \tau)\right\|_{H^{2} \cap H_{0}^{1}(0,1)}^{2} \leqslant C(T-\tau), \quad \text { for all } 0 \leqslant \tau<T,
$$

with a different constant $C(T-\tau)$. 
By extracting subsequences once again (that we still denote by the index $\varepsilon$ ) we deduce that

$$
\begin{aligned}
& \varphi^{\varepsilon}(x, t) \rightarrow \varphi(x, t) \text { strongly in } L^{2}((0,1) \times(0, \tau)), \\
& \varphi^{\varepsilon}(x, \tau) \rightarrow \varphi(x, \tau) \text { strongly in } L^{2}(0,1),
\end{aligned}
$$

for all $0 \leqslant \tau<T$.

Multiplying (104) by an arbitrary function $\theta \in C^{\infty}((0,1) \times(0, \tau))$ such that $\theta(0, t)=$ $\theta(1, t)=\theta(x, 0)=0$ and integrating by parts,

$$
\int_{0}^{\tau} \int_{0}^{1} \varphi^{\varepsilon}\left(\rho\left(\frac{x}{\varepsilon}\right) \theta_{t}-\theta_{x x}\right) \mathrm{d} x \mathrm{~d} t-\int_{0}^{1} \rho\left(\frac{x}{\varepsilon}\right) \varphi^{\varepsilon} \theta(x, \tau) \mathrm{d} x=0 .
$$

Passing to the limit $\varepsilon \rightarrow 0$ in the above expression we obtain that

$$
\int_{0}^{\tau} \int_{0}^{1} \varphi\left(\bar{\rho} \theta_{t}-\theta_{x x}\right) \mathrm{d} x \mathrm{~d} t-\int_{0}^{1} \bar{\rho} \varphi \theta(x, \tau) \mathrm{d} x=0 .
$$

We deduce that $\varphi$ verifies the equation

$$
\begin{cases}\bar{\rho} \varphi_{t}+\varphi_{x x}=0, & 0<x<1,0<t<\tau \\ \varphi(0, t)=\varphi(1, t)=0, & 0<t<\tau\end{cases}
$$

for all $0<\tau<T$.

By (122) and (125) we deduce that

$$
\varphi_{x}^{\varepsilon}(1, t) \rightarrow \varphi_{x}(1, t) \text { strongly in } L^{2}(0, \tau), \quad \text { as } \varepsilon \rightarrow 0,
$$

for all $0<\tau<T$.

Taking (122) and (126) into account we deduce that

$$
\varphi_{x}(1, t)=\varsigma(t) \text { for all } 0<t<T .
$$

In order to identify the function $\varphi(x, t)$ let us consider the adjoint limit system:

$$
\begin{cases}\bar{\rho} \phi_{t}+\phi_{x x}=0, & 0<x<1,0<t<T, \\ \phi(0, t)=\phi(1, t)=0, & 0<t<T \\ \phi(x, T)=\phi^{0}(x), & 0<x<1 .\end{cases}
$$

We define the space

$$
F=\left\{\begin{array}{cl}
g \in L^{2}(0, T): & g=\phi_{x}(1, t) \text { for some solution } \phi \text { of }(128) \\
& \text { with } \phi \in L_{\text {loc }}^{2}\left(0, T ; H^{2} \cap H_{0}^{1}(0,1)\right)
\end{array}\right\}
$$


endowed with the norm

$$
\|g\|_{F}=\left(\int_{0}^{T}\left|\phi_{x}(1, t)\right|^{2} \mathrm{~d} t\right)^{\frac{1}{2}} .
$$

We also define the following quadratic functional on $F$ :

$$
J(\phi)=\frac{1}{2} \int_{0}^{T}\left|\phi_{x}(1, t)\right|^{2} \mathrm{~d} t-\bar{\rho} \int_{0}^{1} u_{0}(x) \phi(x, 0) \mathrm{d} x .
$$

The solution $\phi$ of (128) satisfies the following observability inequality:

$$
\|\phi(\cdot, 0)\|_{L^{2}(0,1)}^{2} \leqslant C \int_{0}^{T}\left|\phi_{x}(1, t)\right|^{2} \mathrm{~d} t,
$$

with $C>0$ independent of the solution $\phi$. This inequatily is well-known to hold, see for instance [14]. It can also be derived as limit when $\varepsilon \rightarrow 0$ of the uniform inequatily (12).

In view of (131) it is easy to see that the functional $J$ achieves its unique minimum at a single point $\psi \in F$, which is characterized by the Euler equation:

$$
\int_{0}^{T} \psi_{x}(1, t) \phi_{x}(1, t) \mathrm{d} t-\int_{0}^{1} \bar{\rho} u_{0}(x) \phi(x, 0) \mathrm{d} x=0,
$$

for all $\phi$ solution of (128).

Comparing (114) and (132), taking into account that $\varsigma(t)=\varphi_{x}(1, t)$, we deduce that

$$
\psi(x, t)=\varphi(x, t)
$$

and consequently

$$
\varphi_{x}^{\varepsilon}(1, t) \rightarrow \psi_{x}(1, t) \text { weakly in } L^{2}(0, T) .
$$

By lower semicontinuity we have that

$$
J(\psi) \leqslant \lim _{\varepsilon \rightarrow 0} \inf \left(J^{\varepsilon}\left(\varphi^{\varepsilon}\right)\right) .
$$

On the other hand, for any $\phi^{0} \in H^{2} \cap H_{0}^{1}(0,1)$, by Lemma 5.1, the solutions of (104) verify that

$$
J(\phi)=\lim _{\varepsilon \rightarrow 0}\left(J^{\varepsilon}\left(\phi^{\varepsilon}\right)\right)
$$

and consequently,

$$
J(\psi) \geqslant \lim _{\varepsilon \rightarrow 0} \sup \left(J^{\varepsilon}\left(\varphi^{\varepsilon}\right)\right) .
$$

By (135), (137) and taking into account that

$$
\varphi^{\varepsilon}(x, 0) \rightarrow \psi(x, 0) \text { strongly in } L^{2}(0,1),
$$


we deduce that

$$
\int_{0}^{T}\left|\varphi_{x}^{\varepsilon}(1, t)\right|^{2} \mathrm{~d} t \rightarrow \int_{0}^{T}\left|\psi_{x}(1, t)\right|^{2} \mathrm{~d} t, \quad \text { as } \varepsilon \rightarrow 0 .
$$

By (134) and (139) we conclude that

$$
\left\{\varphi_{x}^{\varepsilon}(1, t)\right\} \rightarrow \psi_{x}(1, t) \text { strongly in } L^{2}(0, T) .
$$

In what concerns the convergence of solutions $u^{\varepsilon}$, proceeding by transposition and with the aid of Lemma 5.1 and Remark 5.1, it can be easily shown that $u^{\varepsilon}$ is uniformly bounded in $L^{\infty}\left(0, T ; H^{-s}(0,1)\right)$ for all $s>3 / 2$. The weak-* limit in $L^{\infty}\left(0, T ; H^{-s}(0,1)\right)$ of $u^{\varepsilon}$ can be easily seen to be the unique solution of (6) with the limit control $f$ that is defined by transposition as well.

\section{Futher comments and results}

In this section we discuss some variants and extensions of the results of this paper and also some open problems.

\subsection{The case $\rho \in W^{1, \infty}(\mathbb{R})$}

In the case $\rho \in W^{1, \infty}(\mathbb{R})$ the above control strategy in three steps can also be applied. The existence of a uniformly bounded sequence of controls $\left\{f^{\varepsilon}\right\}$ can be proved provided $T>T_{0}$, where $T_{0}$ is a large enough constant.

The case $\rho \in L^{\infty}(\mathbb{R})$ is by now an open problem.

\subsection{Dimension $N>1$}

Using the partial controllability result proved in [13] for the wave equation with rapidly oscillating coefficients and the methods in [19] the partial null controllability result stated in Theorem 3.1 can be extended to dimension $N>1$ when the control acts on a part of the boundary satisfying the geometric control condition for the limit wave equation. However in the case of several space dimensions Lemma 4.1 can not be applied directly since we may not use the change of variables (150)-(160).

One may expect that a convenient Global Carleman Estimate for the adjoint system will provide a result similar to Lemma 4.1 in several space dimensions. This would allow to apply the above control strategy in three steps and to conclude the uniform null-controllability of the heat equation with rapidly oscillating coefficients in several space dimensions and when the control acts on a subset of the boundary that satisfies the geometric control condition for the limit wave equation. But this is by now an open problem. 


\subsection{System $u_{t}-\left(a\left(\frac{x}{\varepsilon}\right) u_{x}\right)_{x}=0$}

Let us consider the following system:

$$
\begin{cases}u_{t}^{\varepsilon}-\left(a\left(\frac{x}{\varepsilon}\right) u_{x}^{\varepsilon}\right)_{x}=0, & 0<x<1,0<t<T, \\ u^{\varepsilon}(0, t)=0, \quad u^{\varepsilon}(1, t)=f^{\varepsilon}(t), & 0<t<T, \\ u^{\varepsilon}(x, 0)=u_{0}(x), & 0<x<1,\end{cases}
$$

where $0<\varepsilon<1$ and $a(x) \in W^{2, \infty}(\mathbb{R})$ is a periodic function satisfying

$$
0<a_{m} \leqslant a(x) \leqslant a_{M}<+\infty,
$$

for all $x \in \mathbb{R}$. Without loss of generality we may assume that $a$ is of period 1 .

The homogenized limit as $\varepsilon \rightarrow 0$ of (141) is the averaged system

$$
\begin{cases}u_{t}-\left(\overline{\left(\frac{1}{a}\right)}\right)^{-1} u_{x x}=0, & 0<x<1,0<t<T, \\ u(0, t)=0, \quad u(1, t)=f(t), & 0<t<T, \\ u(x, 0)=u_{0}(x), & 0<x<1,\end{cases}
$$

where

$$
\overline{\left(\frac{1}{a}\right)}=\int_{0}^{1} \frac{\mathrm{d} s}{a(s)}
$$

(see [5] as a reference on homogenization theory).

We have the following result:

THEOREM 7.1. - Under the above assumptions on $a$, for any $T>0$, and $u_{0} \in$ $L^{2}(0,1)$ there exists a sequence of controls $\left\{f^{\varepsilon}\right\}$ of $(141)$ such that

$$
\left\{f^{\varepsilon}\right\} \rightarrow f \text { strongly in } L^{2}(0, T) \text { as } \varepsilon \rightarrow 0,
$$

$f$ being a control of the limit system (143).

The proof of Theorem 7.1 is based on the following change of variables:

$$
z=\frac{\int_{0}^{x / \varepsilon} \frac{\mathrm{d} s}{a(s)}}{\int_{0}^{1 / \varepsilon} \frac{\mathrm{d} s}{a(s)}}=\delta(\varepsilon) h\left(\frac{x}{\varepsilon}\right)
$$

where

$$
h(t)=\int_{0}^{t} \frac{\mathrm{d} s}{a(s)} \quad \text { and } \quad \delta(\varepsilon)=\frac{1}{\int_{0}^{1 / \varepsilon} \frac{\mathrm{d} s}{a(s)}}=\frac{1}{h(1 / \varepsilon)} .
$$

Then system (141) is equivalent to

$$
\begin{cases}(\varepsilon / \delta(\varepsilon))^{2} a\left(h^{-1}(z / \delta(\varepsilon))\right) u_{t}^{\varepsilon}-u_{z z}^{\varepsilon}=0, & 0<z<1,0<t<T, \\ u^{\varepsilon}(0, t)=0, \quad u^{\varepsilon}(1, t)=f^{\varepsilon}(t), & 0<t<T, \\ u^{\varepsilon}(z, 0)=u_{0}\left(\varepsilon h^{-1}(z / \delta(\varepsilon))\right), & 0<z<1,\end{cases}
$$


where $a\left(h^{-1}(t)\right)$ is periodic, with period,

$$
T=\int_{0}^{1} \frac{1}{a(s)} \mathrm{d} s .
$$

The proof of Theorem 1.1 can be generalized to system (148).

\section{Appendix}

\subsection{Proof of Lemma 4.1}

Let us consider the following change of variables in (72):

$$
y=H(x)=\int_{0}^{x} \sqrt{a(s)} \mathrm{d} s .
$$

Note that $H$ is a monotone increasing function. We have that

$$
x=H^{-1}(y) .
$$

Then

$$
\begin{gathered}
\frac{\mathrm{d}}{\mathrm{d} y}=\frac{1}{\sqrt{a(x)}} \frac{\mathrm{d}}{\mathrm{d} x}, \\
\frac{1}{a(x)} \frac{\mathrm{d}^{2}}{\mathrm{~d} x^{2}}=\frac{1}{a(x)} \frac{\mathrm{d}}{\mathrm{d} x}\left(\sqrt{a(x)} \frac{\mathrm{d}}{\mathrm{d} y}\right)=\frac{a^{\prime}(x)}{2 a(x)^{\frac{3}{2}}} \frac{\mathrm{d}}{\mathrm{d} y}+\frac{\mathrm{d}^{2}}{\mathrm{~d} y^{2}},
\end{gathered}
$$

where $a^{\prime}(x)$ denotes the first derivative of $a$ with respect to $x$.

System (72) is equivalent to

$$
\begin{cases}\phi_{t}+\phi_{y y}+\frac{a^{\prime}\left(H^{-1}(y)\right)}{2 a\left(H^{-1}(y)\right)^{3 / 2}} \phi_{y}=0, & 0<y<y_{1}, 0<t<T, \\ \phi(0, t)=\phi\left(y_{1}, t\right)=0, & 0<t<T, \\ \phi(y, T)=\phi^{0}, & 0<y<y_{1},\end{cases}
$$

where $y_{1}$ is given by

$$
y_{1}=\int_{0}^{1} \sqrt{a(s)} \mathrm{d} s .
$$

Note that

$$
\sqrt{a_{m}} \leqslant y_{1} \leqslant \sqrt{a_{M}}
$$

The function $\theta(y, t)$ given by

$$
\theta(y, t)=\mathrm{e}^{\int_{0}^{y} a^{\prime}\left(H^{-1}(s)\right) / 4 a\left(H^{-1}(s)\right)^{3 / 2} \mathrm{~d} s} \phi(y, t),
$$


verifies

$$
\begin{cases}\theta_{t}+\theta_{y y}-b(y) \theta=0, & 0<y<y_{1}, 0<t<T \\ \theta(0, t)=\theta\left(y_{1}, t\right)=0, & 0<t<T \\ \theta(y, T)=\theta^{0}, & 0<y<y_{1}\end{cases}
$$

where

$$
b(y)=\left(\left(\frac{a^{\prime}\left(H^{-1}(y)\right)}{4 a\left(H^{-1}(y)\right)^{3 / 2}}\right)^{\prime}+\left(\frac{a^{\prime}\left(H^{-1}(y)\right)}{4 a\left(H^{-1}(y)\right)^{3 / 2}}\right)^{2}\right),
$$

and

$$
\theta^{0}=\mathrm{e}^{-\int_{0}^{y} a^{\prime}\left(H^{-1}(s)\right) / 4 a\left(H^{-1}(s)\right)^{3 / 2} \mathrm{~d} s} \phi^{0} .
$$

We have the following observability result for the solutions of (158).

LEMMA 8.1. - There exist positive constants $C_{1}$ and $C_{2}$, independent of the function $b(y)$, such that

$$
\|\theta(\cdot, 0)\|_{L^{2}\left(0, y_{1}\right)}^{2} \leqslant C_{1} \exp \left\{C_{2}\|b\|_{L^{\infty}\left(0, y_{1}\right)}^{2 / 3}+\int_{0}^{y_{1}}\left|\frac{a^{\prime}\left(H^{-1}(s)\right)}{2 a\left(H^{-1}(s)\right)^{3 / 2}}\right| \mathrm{d} s\right\} \int_{0}^{T}\left|\theta_{y}\left(y_{1}, t\right)\right|^{2} \mathrm{~d} t,
$$

for all $\theta$ solution of (158).

Applying Lemma 8.1 with $b(y)$ given by (159) and taking (150), (152) and (157) into account we obtain (74).

It only remains to prove Lemma 8.1.

Proof of Lemma 8.1. - Let us consider the space

$$
Z_{0}=\left\{q \in C^{2}\left(\left[0, y_{1}\right] \times[0, T]\right): q=0 \text { in }\left\{0, y_{1}\right\} \times(0, T)\right\},
$$

where $y_{1}$ is given by (155). In the appendix we prove the following result, see also [10] and [11]:

LEMMA 8.2. - There exist a positive function $\xi \in C^{4}\left(\left[0, y_{1}\right] \times(0, T)\right)$ and positive constants $s_{0}, s_{1}$ and $C_{*}$ which depend on $T$ and $y_{1}$, such that:

$$
\begin{aligned}
& \frac{1}{s} \int_{\left(0, y_{1}\right) \times(0, T)} \xi^{-2 s} t(T-t)\left(\left|\partial_{t} q\right|^{2}+\left|\partial_{y y} q\right|^{2}\right)+s \int_{\left(0, y_{1}\right) \times(0, T)} \frac{\xi^{-2 s}}{t(T-t)}\left|\partial_{y} q\right|^{2} \\
& \quad+s^{3} \int_{\left(0, y_{1}\right) \times(0, T)} \frac{\xi^{-2 s}}{t^{3}(T-t)^{3}}|q|^{2} \\
& \leqslant C_{*}\left(\int_{\left(0, y_{1}\right) \times(0, T)} \xi^{-2 s}\left|\partial_{t} q+\partial_{y y} q-b(y, t) q\right|^{2}+s \int_{0}^{T} \frac{\xi^{-2 s}}{t(T-t)}\left|q_{y}\left(y_{1}, t\right)\right|^{2}\right),
\end{aligned}
$$

holds for all $b \in L^{\infty}\left(\left(0, y_{1}\right) \times(0, T)\right), s \geqslant s_{0}+s_{1}\|b\|_{\infty}^{2 / 3}$ and $q \in Z_{0}$. 
Remark 8.1. - Taking into account that

$$
0<\sqrt{a_{m}} \leqslant y_{1} \leqslant \sqrt{a_{M}}<+\infty,
$$

the constant $C_{*}$ in (163) can be chosen to be independent of $y_{1}$. See the proof of Lemma 8.2.

Inequality (163) is known as Global Carleman Inequality associated to the system

$$
\begin{cases}q_{t}+q_{y y}-b(y, t) q=0, & 0<y<y_{1}, 0<t<T, \\ q(0, t)=q\left(y_{1}, t\right)=0, & 0<t<T, \\ q(y, T)=q_{0}, & 0<y<y_{1} .\end{cases}
$$

The function $\xi$ for which (163) holds is not unique. One possible choice for $\xi$ is the following:

$$
\xi(y, t)=\mathrm{e}^{\frac{\mathrm{e}^{\lambda \bar{\xi}}-\mathrm{e}^{\lambda \xi_{0}}}{t(T-t)}}
$$

where $\lambda$ is a large enough constant, independent of $b, \bar{\xi}$ is a constant such that

$$
\bar{\xi}>\xi_{0}(y)
$$

for all $y \in\left(0, y_{1}\right)$ and $\xi_{0}(y)$ is an special function. In our case, i.e. in one space dimension, any $C^{4}$ strictly increasing function is a suitable choice (we refer to [10] and [11] for the construction of this function in several space dimensions).

Applying (163) to the solutions of (158) and by a density argument we have that

$$
s^{2} \int_{\left(0, y_{1}\right) \times(0, T)} \frac{\xi^{-2 s}}{t^{3}(T-t)^{3}}|\theta|^{2} \leqslant C_{*} \int_{0}^{T} \frac{\xi^{-2 s}}{t(T-t)}\left|\theta_{y}\left(y_{1}, t\right)\right|^{2},
$$

for all $s \geqslant s_{0}+s_{1}\|b\|_{\infty}^{2 / 3}$. The function

$$
\frac{\xi^{-2 s}}{t(T-t)}=\frac{\mathrm{e}^{-2 s \frac{\mathrm{e}^{\lambda \bar{\xi}_{-}} \mathrm{e} \mathrm{e}_{0}}{t(T-t)}}}{t(T-t)}
$$

is uniformly bounded (note that $\bar{\xi}>\xi_{0}(y)$ ) and then

$$
\int_{0}^{T} \frac{\xi^{-2 s}}{t(T-t)}\left|\partial_{y} \theta\left(y_{1}, t\right)\right|^{2} \leqslant C \int_{0}^{T}\left|\partial_{y} \theta\left(y_{1}, t\right)\right|^{2},
$$

where $C$ is a positive constant which depends only on $T$.

On the other hand,

$$
\frac{\xi^{-2 s}}{t^{3}(T-t)^{3}}=\frac{\mathrm{e}^{-s \frac{\mathrm{e}^{\lambda \bar{\xi}}-\mathrm{e}^{\lambda \xi} \xi_{0}}{t(T-t)}}}{t^{3}(T-t)^{3}} \geqslant \frac{\mathrm{e}^{-2 s \frac{\mathrm{e}^{\bar{\xi}} \bar{\xi}}{t(T-t)}}}{t^{3}(T-t)^{3}}
$$


and then,

$$
\int_{0}^{T} \int_{0}^{y_{1}} \frac{\xi^{-2 s}}{t^{3}(T-t)^{3}}|\theta(y, t)|^{2} \mathrm{~d} y \mathrm{~d} t \geqslant \int_{0}^{T} \frac{\mathrm{e}^{-2 s \frac{\mathrm{e}^{\lambda \bar{\xi}}}{t(T-t)}}}{t^{3}(T-t)^{3}} \int_{0}^{y_{1}}|\theta(y, t)|^{2} \mathrm{~d} y \mathrm{~d} t .
$$

We have the following estimate for the solutions of (158):

LEMMA 8.3. - There exists $C>0$ independent of a such that

$$
\|\theta(\cdot, t)\|_{L^{2}\left(0, y_{1}\right)}^{2} \geqslant C \mathrm{e}^{-\int_{0}^{y_{1}}\left|a^{\prime}\left(H^{-1}(s)\right) / 2 a\left(H^{-1}(s)\right)^{2 / 3}\right| \mathrm{d} s}\|\theta(\cdot, 0)\|_{L^{2}\left(0, y_{1}\right)}^{2},
$$

for all $t \geqslant 0$ and $\theta$ solution of (158).

Proof. - Assume that $T>0$ is fixed. Multiplying (72) by $\phi$ and integrating in space we get

$$
\frac{1}{2} \frac{\mathrm{d}}{\mathrm{d} t} \int_{0}^{1} a(x)|\phi|^{2} \mathrm{~d} x-\int_{0}^{1}\left|\phi_{x}\right|^{2} \mathrm{~d} x=0
$$

We deduce that

$$
\frac{\mathrm{d}}{\mathrm{d} t} \int_{0}^{1} a(x)|\phi|^{2} \mathrm{~d} x \geqslant 0
$$

Consequently,

$$
\int_{0}^{1} a(x)\left|\phi^{0}\right|^{2} \mathrm{~d} x \geqslant \int_{0}^{1} a(x)|\phi(x, 0)|^{2} \mathrm{~d} x .
$$

By (150) and (157) we obtain that

$$
\begin{aligned}
& \int_{0}^{y_{1}} \sqrt{a\left(H^{-1}(y)\right)}\left|\mathrm{e}^{\int_{0}^{y} a^{\prime}\left(H^{-1}(s)\right) / 4 a\left(H^{-1}(s)\right)^{3 / 2} \mathrm{~d} s} \theta^{0}\right|^{2} \mathrm{~d} y \\
& \geqslant \int_{0}^{y_{1}} \sqrt{a\left(H^{-1}(y)\right)}\left|\mathrm{e}^{-\int_{0}^{y} a^{\prime}\left(H^{-1}(s)\right) / 4 a\left(H^{-1}(s)\right)^{2 / 3} \mathrm{~d} s} \theta(y, 0)\right|^{2} \mathrm{~d} y .
\end{aligned}
$$

From (176) we obtain (172).

Going back to (171) we have that

$$
\begin{aligned}
& \int_{0}^{T} \int_{0}^{y_{1}} \frac{\xi^{-2 s}}{t^{3}(T-t)^{3}}|\theta(y, t)|^{2} \\
& \quad \geqslant C \mathrm{e}^{-\int_{0}^{y_{1}}\left|\int_{0}^{y} a^{\prime}\left(H^{-1}(s)\right) / 4 a\left(H^{-1}(s)\right)^{3 / 2}\right| \mathrm{d} s} \int_{0}^{y_{1}}|\theta(y, 0)|^{2} \mathrm{~d} y \int_{0}^{T} \frac{\mathrm{e}^{-2 s \frac{\mathrm{e}^{\lambda \bar{\xi}}}{t^{T}(T-t)}}}{t^{3}(T-t)^{3}} \mathrm{~d} t,
\end{aligned}
$$


and then, by (169),

$$
\begin{aligned}
& \int_{0}^{y_{1}}|\theta(y, 0)|^{2} \mathrm{~d} y \int_{0}^{T} \frac{\mathrm{e}^{-2 s \frac{e^{\lambda \bar{\xi}}}{T(T-t)}}}{t^{3}(T-t)^{3}} \mathrm{~d} t \\
& \quad \leqslant C \mathrm{e}^{\int_{0}^{y_{1}}\left|\int_{0}^{y} a^{\prime}\left(H^{-1}(s)\right) / 4 a\left(H^{-1}(s)\right)^{2 / 3}\right| \mathrm{d} s} \int_{0}^{T}\left|\partial_{y} \theta\left(y_{1}, t\right)\right|^{2} \mathrm{~d} t,
\end{aligned}
$$

for all $s \geqslant s_{0}+s_{1}\|b\|_{\infty}^{2 / 3}$. Let us take in (178)

$$
s=s_{0}+s_{1}\|b\|_{\infty}^{2 / 3} .
$$

We have that

$$
\int_{0}^{T} \frac{\mathrm{e}^{-2 s \frac{\mathrm{e}^{\lambda \bar{\xi}}}{t(T-t)}}}{t^{3}(T-t)^{3}} \mathrm{~d} t \geqslant \int_{\frac{T}{4}}^{\frac{3 T}{4}} \frac{\mathrm{e}^{-2 s_{0}} \frac{\mathrm{e}^{\lambda \bar{\xi}}}{t(T-t)}}{t^{3}(T-t)^{3}} \mathrm{e}^{-2 s_{1}\|b\|_{\infty}^{2 / 3} \frac{\mathrm{e}^{\lambda \bar{\xi}}}{t(T-t)}} \mathrm{d} t \geqslant C_{1}(T) \mathrm{e}^{-C_{2}(T)\|b\|_{\infty}^{2 / 3}} .
$$

From (178) and (180) we obtain (161).

\subsection{Proof of Lemma 8.2}

The main ideas for this proof are presented in [11] and [10]. In this section we give a sketch of the proof of the inequality we need.

We are going to prove inequality (163) with $y_{1}=1$. In fact, after carefully analyzing the proof below it can be seen that the constant $C_{*}$ depends continously on $y_{1}$. From this result and taking into account that

$$
0<\sqrt{a_{m}} \leqslant y_{1} \leqslant \sqrt{a_{M}}<+\infty,
$$

it can be proved that inequality (163) holds with a positive constant $C_{*}$ depending only on $a_{m}, a_{M}$ and $T$.

In this section $\|\cdot\|$ and $(\cdot, \cdot)$ will stand for the norm and the scalar product in $L^{2}((0,1) \times(0, T))$, respectively. Futhermore, all integrals below are extended to $(0,1) \times(0, T)$ unless otherwise specified.

Let us start with the case $b=0$. We define the following functions:

$$
\xi_{1}(x)=\mathrm{e}^{\lambda \bar{\xi}}-\mathrm{e}^{\lambda \xi_{0}(x)}, \quad \varphi(x, t)=\frac{\xi_{1}(x)}{t(T-t)} \quad \text { and } \quad \xi(x, t)=\mathrm{e}^{\varphi(x, t)},
$$

where $\lambda$ is a positive constant that we will fix later, $\xi_{0}(x)$ is a positive function in $C^{4}[0,1]$ with $\left(\xi_{0}\right)_{x} \neq 0$ for all $x \in[0,1]$ and $\left.\left(\xi_{0}\right)_{x}\right|_{x=0} \geqslant 0$. Moreover $\bar{\xi}$ is a positive constant such that $\bar{\xi}>\xi_{0}(x)$ for all $x \in[0,1]$.

Let us consider $q \in Z_{0}$. We denote

$$
\psi=\xi^{-s} q=\mathrm{e}^{-s \varphi} q
$$


where $s$ is a parameter which will be chosen later. We have that

$$
\begin{aligned}
\psi_{t}+\psi_{x x}= & -s \psi \varphi_{t}+\mathrm{e}^{-s \varphi} q_{t} \\
& -s \psi_{x} \varphi_{x}-s \psi \varphi_{x x}-s \psi_{x} \varphi_{x}-s^{2} \psi\left(\varphi_{x}\right)^{2}+\mathrm{e}^{-s \varphi} q_{x x} .
\end{aligned}
$$

We introduce the notation

$$
\begin{gathered}
M_{1}(\psi)=\psi_{t}+2 s \psi_{x} \varphi_{x}, \\
M_{2}(\psi)=\psi_{x x}+s^{2} \psi\left(\varphi_{x}\right)^{2}+s \psi \varphi_{t}, \\
w_{s}=\mathrm{e}^{-s \varphi}\left(q_{t}+q_{x x}\right),
\end{gathered}
$$

so that

$$
M_{1}(\psi)+M_{2}(\psi)=w_{s}-s \psi \varphi_{x x} .
$$

We deduce the following identity,

$$
\left\|M_{1}(\psi)\right\|^{2}+\left\|M_{2}(\psi)\right\|^{2}+2\left(M_{1}(\psi), M_{2}(\psi)\right)=\left\|w_{s}-s \psi \varphi_{x x}\right\|^{2} .
$$

Now, let us analyze the scalar product in (189):

$$
\begin{aligned}
\left(M_{1}(\psi), M_{2}(\psi)\right)= & \int \psi_{t} \psi_{x x}+2 s \int \psi_{x} \varphi_{x} \psi_{x x}+s^{2} \int \psi \psi_{t}\left(\varphi_{x}\right)^{2} \\
& +2 s^{3} \int \psi_{x} \varphi_{x} \psi\left|\varphi_{x}\right|^{2}+s \int \psi_{t} \varphi_{t} \psi+2 s^{2} \int \psi \varphi_{x} \psi_{x} \varphi_{t} \\
= & I_{1}+2 s I_{2}+s^{2} I_{3}+2 s^{3} I_{4}+s I_{5}+2 s^{2} I_{6} .
\end{aligned}
$$

We have that

$$
\begin{aligned}
I_{1} & =\int \psi_{t} \psi_{x x}=-\int\left(\psi_{x}\right)_{t} \psi_{x}=-\frac{1}{2} \int\left(\left|\psi_{x}\right|^{2}\right)_{t}=0, \\
I_{2} & =\int \psi_{x} \varphi_{x} \psi_{x x}=\frac{1}{2} \int\left(\left|\psi_{x}\right|^{2}\right)_{x} \varphi_{x} \\
& =-\frac{1}{2} \int \varphi_{x x}\left|\psi_{x}\right|^{2}+\frac{1}{2} \int_{0}^{T} \varphi_{x}(1, t)\left|\psi_{x}(1, t)\right|^{2}-\frac{1}{2} \int_{0}^{T} \varphi_{x}(0, t)\left|\psi_{x}(0, t)\right|^{2}, \\
I_{3} & =\int \psi \psi_{t}\left(\varphi_{x}\right)^{2}=\frac{1}{2} \int\left(|\psi|^{2}\right)_{t}\left(\varphi_{x}\right)^{2}=-\frac{1}{2} \int|\psi|^{2}\left(\left|\varphi_{x}\right|^{2}\right)_{t}, \\
I_{4} & =\int \psi_{x} \varphi_{x} \psi\left|\varphi_{x}\right|^{2}=\frac{1}{2} \int\left(|\psi|^{2}\right)_{x}\left(\varphi_{x}\right)^{3}=-\frac{3}{2} \int|\psi|^{2}\left|\varphi_{x}\right|^{2} \varphi_{x x}, \\
I_{5} & =\int \psi_{t}\left(\varphi_{t}\right) \psi=\frac{1}{2} \int\left(|\psi|^{2}\right)_{t} \varphi_{t}=-\frac{1}{2} \int|\psi|^{2} \varphi_{t t},
\end{aligned}
$$

and finally,

$$
I_{6}=\int \psi \varphi_{x} \psi_{x} \varphi_{t}=\frac{1}{2} \int\left(|\psi|^{2}\right)_{x} \varphi_{x} \varphi_{t}=-\frac{1}{2} \int|\psi|^{2}\left(\varphi_{x x} \varphi_{t}+\varphi_{x}\left(\varphi_{x}\right)_{t}\right) .
$$


Consequently,

$$
\begin{aligned}
& \left\|M_{1}(\psi)\right\|^{2}+\left\|M_{2}(\psi)\right\|^{2}-6 s^{3} \int|\psi|^{2}\left|\varphi_{x}\right|^{2} \varphi_{x x} \\
& \quad+2 s \int_{0}^{T} \varphi_{x}(1, t)\left|\psi_{x}(1, t)\right|^{2}-2 s \int \varphi_{x x}\left|\psi_{x}\right|^{2}-2 s \int_{0}^{T} \varphi_{x}(0, t)\left|\psi_{x}(0, t)\right|^{2} \\
& =\left\|w_{s}-s \psi \varphi_{x x}\right\|^{2}+s \int|\psi|^{2} \varphi_{t t}+2 s^{2} \int|\psi|^{2}\left(\varphi_{x x} \varphi_{t}+2 \varphi_{x}\left(\varphi_{x}\right)_{t}\right) .
\end{aligned}
$$

First note that

$$
-2 s \int_{0}^{T} \varphi_{x}(0, t)\left|\psi_{x}(0, t)\right|^{2} \geqslant 0,
$$

and then

$$
\begin{aligned}
& \left\|M_{1}(\psi)\right\|^{2}+\left\|M_{2}(\psi)\right\|^{2}-6 s^{3} \int|\psi|^{2}\left|\varphi_{x}\right|^{2} \varphi_{x x} \\
& \quad+2 s \int_{0}^{T} \varphi_{x}(1, t)\left|\psi_{x}(1, t)\right|^{2}-2 s \int \varphi_{x x}\left|\psi_{x}\right|^{2} \\
& \leqslant\left\|w_{s}-s \psi \varphi_{x x}\right\|+s \int|\psi|^{2} \varphi_{t t}+2 s^{2} \int|\psi|^{2}\left(\varphi_{x x} \varphi_{t}+2 \varphi_{x}\left(\varphi_{x}\right)_{t}\right) .
\end{aligned}
$$

Using the particular form of $\varphi$ we see that for $\lambda$ large enough

$$
-\left|\varphi_{x}\right|^{2} \varphi_{x x} \geqslant A \lambda\left|\varphi_{x}\right|^{3}+A\left|\varphi_{x}\right|^{3},
$$

where $A$ is a constant independent of $T$. On the other hand, we can see that

$$
\left|\varphi_{t t}\right|+\left|\varphi_{x}\left(\varphi_{x}\right)_{t}\right|+\left|\varphi_{x x} \varphi_{t}\right| \leqslant C\left|\varphi_{x}\right|^{3},
$$

where $C$ is a positive constant.

Consequently,

$$
s \int|\psi|^{2} \varphi_{t t}+2 s^{2} \int|\psi|^{2}\left(\varphi_{x x} \varphi_{t}+2 \varphi_{x}\left(\varphi_{x}\right)_{t}\right) \leqslant C s^{2} \int\left|\varphi_{x}\right|^{3}|\psi|^{2} .
$$

Then,

$$
\begin{aligned}
& \left\|M_{1}(\psi)\right\|^{2}+\left\|M_{2}(\psi)\right\|^{2}+6 s^{3} \int|\psi|^{2}\left(A \lambda\left|\varphi_{x}\right|^{3}+A\left|\varphi_{x}\right|^{3}\right)+2 s \int_{0}^{T} \varphi_{x}(1, t)\left|\psi_{x}(1, t)\right|^{2} \\
& -2 s \int \varphi_{x x}\left|\psi_{x}\right|^{2} \leqslant\left\|w_{s}-s \psi \varphi_{x x}\right\|^{2}+C s^{2} \int\left|\varphi_{x}\right|^{3}|\psi|^{2}
\end{aligned}
$$

and 


$$
\begin{aligned}
& \left\|M_{1}(\psi)\right\|^{2}+\left\|M_{2}(\psi)\right\|^{2}+6 s^{3} \int|\psi|^{2}\left(A \lambda\left|\varphi_{x}\right|^{3}+A\left|\varphi_{x}\right|^{3}\right)-C s^{2} \int\left|\varphi_{x}\right|^{3}|\psi|^{2} \\
& \quad+2 s \int_{0}^{T} \varphi_{x}(1, t)\left|\psi_{x}(1, t)\right|^{2}-2 s \int \varphi_{x x}\left|\psi_{x}\right|^{2} \leqslant\left\|w_{s}-s \psi \varphi_{x x}\right\|^{2} .
\end{aligned}
$$

For $s$ large enough,

$$
\begin{aligned}
& \left\|M_{1}(\psi)\right\|^{2}+\left\|M_{2}(\psi)\right\|^{2}+s^{3} \int|\psi|^{2}\left(A \lambda\left|\varphi_{x}\right|^{3}+A\left|\varphi_{x}\right|^{3}\right)-2 s \int \varphi_{x x}\left|\psi_{x}\right|^{2} \\
& \quad \leqslant\left\|w_{s}-s \psi \varphi_{x x}\right\|^{2}-2 s \int_{0}^{T} \varphi_{x}(1, t)\left|\psi_{x}(1, t)\right|^{2} .
\end{aligned}
$$

Using that

$$
\left\|w_{s}-s \psi \varphi_{x x}\right\|^{2} \leqslant 2\left\|w_{s}\right\|^{2}+2 s^{2} \int\left|\varphi_{x x}\right|^{2}|\psi|^{2}
$$

we obtain that

$$
\begin{aligned}
& \left\|M_{1}(\psi)\right\|^{2}+\left\|M_{2}(\psi)\right\|^{2}+s^{3} \int|\psi|^{2}\left(A \lambda\left|\varphi_{x}\right|^{3}+A\left|\varphi_{x}\right|^{3}\right)-2 s \int \varphi_{x x}\left|\psi_{x}\right|^{2} \\
& \leqslant 2\left\|w_{s}\right\|^{2}+2 s^{2} \int\left|\varphi_{x x}\right|^{2}|\psi|^{2}-2 s \int_{0}^{T} \varphi_{x}(1, t)\left|\psi_{x}(1, t)\right|^{2} .
\end{aligned}
$$

Using once again that $s$ is sufficiently large,

$$
2 s^{2}\left|\varphi_{x x}\right|^{2} \leqslant A \lambda s^{3}\left|\varphi_{x}\right|^{3}\left|\varphi_{x}\right|^{3} .
$$

The term $2 s^{2} \int\left|\varphi_{x x}\right|^{2}|\psi|^{2}$ in (207) is dominated by $s^{3} \int|\psi|^{2} A \lambda\left|\varphi_{x}\right|^{3}$. Then

$$
\begin{gathered}
\left\|M_{1}(\psi)\right\|^{2}+\left\|M_{2}(\psi)\right\|^{2}+s^{3} \int|\psi|^{2}\left(A \lambda\left|\varphi_{x}\right|^{3}+A\left|\varphi_{x}\right|^{3}\right) \\
\leqslant 2\left\|w_{s}\right\|^{2}+2 s \int \varphi_{x x}\left|\psi_{x}\right|^{2}-2 s \int_{0}^{T} \varphi_{x}(1, t)\left|\psi_{x}(1, t)\right|^{2} .
\end{gathered}
$$

On the other hand, integrating by parts,

$$
\begin{aligned}
2 s \int \varphi_{x x}\left|\psi_{x}\right|^{2} & =2 s \int \varphi_{x x} \psi_{x} \psi_{x}=-2 s \int \psi \varphi_{x x x} \psi_{x}-2 s \int \psi \varphi_{x x} \psi_{x x} \\
& =-s \int \varphi_{x x x}\left(|\psi|^{2}\right)_{x}-2 s \int \psi \varphi_{x x} \psi_{x x} \\
& =s \int \varphi_{x x x x}|\psi|^{2}-2 s \int \psi \varphi_{x x} \psi_{x x} .
\end{aligned}
$$

By (188),

$$
-2 s \int \psi \varphi_{x x} \psi_{x x}=\int 2 s\left(M_{1}(\psi)+s^{2} \psi\left(\varphi_{x}\right)^{2}+s \psi \varphi_{t}-w_{s}+s \psi \varphi_{x x}\right) \psi \varphi_{x x} .
$$


Taking $\lambda$ large enough we have that $\varphi_{x x} \leqslant 0$, and

$$
\begin{aligned}
-2 s \int \psi \varphi_{x x} \psi_{x x} & \leqslant \int 2 s\left(M_{1}(\psi)+s \psi \varphi_{t}-w_{s}+s \psi \varphi_{x x}\right) \psi \varphi_{x x} \\
& \leqslant \frac{1}{2}\left\|M_{1}(\psi)\right\|^{2}+\left\|w_{s}\right\|^{2}+C s^{2} \int\left(\varphi_{x x} \varphi_{t}+\left|\varphi_{x x}\right|^{2}\right)|\psi|^{2} .
\end{aligned}
$$

By (209),

$$
\begin{aligned}
& \left\|M_{1}(\psi)\right\|^{2}+\left\|M_{2}(\psi)\right\|^{2}+s^{3} \int|\psi|^{2}\left(A \lambda\left|\varphi_{x}\right|^{3}+A\left|\varphi_{x}\right|^{3}\right) \\
& \leqslant 2\left\|w_{s}\right\|^{2}+2 s \int \varphi_{x x}\left|\psi_{x}\right|^{2}-2 s \int_{0}^{T} \varphi_{x}(1, t)\left|\psi_{x}(1, t)\right|^{2} \\
& =2\left\|w_{s}\right\|^{2}+s \int \varphi_{x x x x}|\psi|^{2}-2 s \int \psi \varphi_{x x} \psi_{x x}-2 s \int_{0}^{T} \varphi_{x}(1, t)\left|\psi_{x}(1, t)\right|^{2} \\
& \leqslant 3\left\|w_{s}\right\|^{2}+s \int \varphi_{x x x x}|\psi|^{2}+\frac{1}{2}\left\|M_{1}(\psi)\right\|^{2} \\
& +C s^{2} \int\left(\varphi_{x x} \varphi_{t}+\left|\varphi_{x x}\right|^{2}\right)|\psi|^{2}-2 s \int_{0}^{T} \varphi_{x}(1, t)\left|\psi_{x}(1, t)\right|^{2} .
\end{aligned}
$$

It can be proved that

$$
s \int \varphi_{x x x x}|\psi|^{2} \leqslant C s^{2} \int\left|\varphi_{x}\right|^{3}|\psi|^{2} .
$$

By (201), (208) and (214) the terms $s \int \varphi_{x x x x}|\psi|^{2}$ and $C s^{2} \int\left(\varphi_{x x} \varphi_{t}+\left|\varphi_{x x}\right|^{2}\right)|\psi|^{2}$ are dominated by

$$
s^{3} \int|\psi|^{2}\left(A \lambda\left|\varphi_{x}\right|^{3}+A\left|\varphi_{x}\right|^{3}\right)
$$

Then

$$
\begin{aligned}
& \left\|M_{1}(\psi)\right\|^{2}+\left\|M_{2}(\psi)\right\|^{2}+s^{3} \int|\psi|^{2}\left(A \lambda\left|\varphi_{x}\right|^{3}+A\left|\varphi_{x}\right|^{3}\right) \\
& \leqslant C\left\|w_{s}\right\|^{2}-2 s \int_{0}^{T} \varphi_{x}(1, t)\left|\psi_{x}(1, t)\right|^{2} .
\end{aligned}
$$

On the other hand,

$$
\begin{aligned}
& \frac{1}{s} \int\left|\varphi_{x}\right|^{-1}\left|\psi_{x x}\right|^{2}=\frac{1}{s} \int\left|\varphi_{x}\right|^{-1}\left(M_{2}(\psi)-s^{2} \psi\left(\varphi_{x}\right)^{2}-s \psi \varphi_{t}\right)^{2} \\
& \quad \leqslant \frac{2}{s} \int\left|\varphi_{x}\right|^{-1}\left|M_{2}(\psi)\right|^{2}+\frac{2}{s} \int\left|\varphi_{x}\right|^{-1}\left(s^{2} \psi\left(\varphi_{x}\right)^{2}-s \psi \varphi_{t}\right)^{2} \\
& \quad \leqslant \frac{2}{s} \int\left|\varphi_{x}\right|^{-1}\left|M_{2}(\psi)\right|^{2}+4 s^{3} \int\left|\varphi_{x}\right|^{3}|\psi|^{2}+4 s \int\left|\varphi_{x}\right|^{-1}\left(\varphi_{t}\right)^{2}|\psi|^{2} .
\end{aligned}
$$


We have that

$$
\frac{2}{s}\left|\varphi_{x}\right|^{-1} \leqslant 1
$$

and

$$
4 s\left|\varphi_{x}\right|^{-1}\left(\varphi_{t}\right)^{2} \leqslant C s^{3}\left|\varphi_{x}\right|^{3},
$$

for $s$ large enough. Consequently,

$$
\frac{2}{s} \int\left|\varphi_{x}\right|^{-1}\left|M_{2}(\psi)\right|^{2} \leqslant\left\|M_{2}(\psi)\right\|^{2}
$$

and by (217),

$$
\frac{1}{s} \int\left|\varphi_{x}\right|^{-1}\left|\psi_{x x}\right|^{2} \leqslant\left\|M_{2}(\psi)\right\|^{2}+C s^{3} \int\left|\varphi_{x}\right|^{3}|\psi|^{2} .
$$

We have that

$$
\begin{aligned}
& \left\|M_{1}(\psi)\right\|^{2}+A \lambda s^{3} \int|\psi|^{2}\left|\varphi_{x}\right|^{3}+\frac{C}{s} \int\left|\varphi_{x}\right|^{-1}\left|\psi_{x x}\right|^{2} \\
& \leqslant C\left\|w_{s}\right\|^{2}-2 s \int_{0}^{T} \varphi_{x}(1, t)\left|\psi_{x}(1, t)\right|^{2} .
\end{aligned}
$$

Taking into account that

$$
A s \lambda^{1 / 2} \int\left|\varphi_{x}\right|\left|\psi_{x}\right|^{2} \leqslant \frac{1}{s} \int\left|\varphi_{x}\right|^{-1}\left|\psi_{x x}\right|^{2}+s^{3} \lambda \int\left|\varphi_{x}\right|^{3}|\psi|^{2},
$$

and

$$
\left|\varphi_{x}\right|=-\varphi_{x},
$$

we get

$$
\left(\left|\varphi_{x}\right|\right)_{x x}=-\varphi_{x x x} .
$$

It can be seen that

$$
-\varphi_{x x x} \leqslant C\left|\varphi_{x}\right|^{3},
$$

and

$$
\frac{s^{3} \lambda}{2} \int\left|\varphi_{x}\right|^{2}|\psi|^{2}+\frac{s \lambda^{1 / 2}}{2} \int\left(\left|\varphi_{x}\right|\right)_{x x}|\psi|^{2} \leqslant s^{3} \lambda \int\left|\varphi_{x}\right|^{3}|\psi|^{2} .
$$

Then

$$
\begin{aligned}
& \left\|M_{1}(\psi)\right\|^{2}+s \lambda^{1 / 2} \int\left|\varphi_{x}\right|\left|\psi_{x}\right|^{2}+\frac{C}{s} \int\left|\varphi_{x}\right|^{-1}\left|\psi_{x x}\right|^{2}+s^{3} \lambda \int\left|\varphi_{x}\right|^{3}|\psi|^{2} \\
& \leqslant C\left\|w_{s}\right\|^{2}-2 s \int_{0}^{T} \varphi_{x}(1, t)\left|\psi_{x}(1, t)\right|^{2} .
\end{aligned}
$$


Finally,

$$
\frac{1}{s} \int\left|\varphi_{x}\right|^{-1}\left|\psi_{t}\right|^{2} \leqslant \frac{2}{s} \int\left|\varphi_{x}\right|^{-1}\left(M_{1}(\psi)\right)^{2}+8 s \int\left|\varphi_{x}\right|\left|\psi_{x}\right|^{2} .
$$

By (218) and, see (223),

$$
C s \int\left|\varphi_{x}\right|\left|\psi_{x}\right|^{2} \leqslant \frac{1}{\lambda^{1 / 2} s} \int\left|\varphi_{x}\right|^{-1}\left|\psi_{x x}\right|^{2}+s^{3} \lambda^{1 / 2} \int\left|\varphi_{x}\right|^{3}|\psi|^{2} .
$$

We obtain that

$$
\frac{1}{s} \int\left|\varphi_{x}\right|^{-1}\left|\psi_{t}\right|^{2} \leqslant\left\|M_{1}(\psi)\right\|^{2}+\frac{1}{\lambda^{1 / 2} s} \int\left|\varphi_{x}\right|^{-1}\left|\psi_{x x}\right|^{2}+s^{3} \lambda^{1 / 2} \int\left|\varphi_{x}\right|^{3}|\psi|^{2} .
$$

Coming back to (228),

$$
\begin{aligned}
& \frac{1}{s} \int\left|\varphi_{x}\right|^{-1}\left|\psi_{t}\right|^{2}+s \lambda^{1 / 2} \int\left|\varphi_{x}\right|\left|\psi_{x}\right|^{2}+\frac{\left(C-\frac{1}{\lambda^{1 / 2}}\right)}{s} \int\left|\varphi_{x}\right|^{-1}\left|\psi_{x x}\right|^{2}+s^{3} \lambda \int\left|\varphi_{x}\right|^{3}|\psi|^{2} \\
& \quad \leqslant C\left\|w_{s}\right\|^{2}-2 s \int_{0}^{T} \varphi_{x}(1, t)\left|\psi_{x}(1, t)\right|^{2}
\end{aligned}
$$

and then

$$
\begin{aligned}
& \frac{1}{s} \int\left|\varphi_{x}\right|^{-1}\left(\left|\psi_{t}\right|^{2}+\left|\psi_{x x}\right|^{2}\right)+s \int\left|\varphi_{x}\right|\left|\psi_{x}\right|^{2}+s^{3} \int\left|\varphi_{x}\right|^{3}|\psi|^{2} \\
& \quad \leqslant C\left(\left\|w_{s}\right\|^{2}-s \int_{0}^{T} \varphi_{x}(1, t)\left|\psi_{x}(1, t)\right|^{2}\right) .
\end{aligned}
$$

Replacing $\psi$ by $\xi^{-s} q$ it is not difficult to deduce that there exists a positive constant $C_{*}$ such that for $\lambda$ and $s$ sufficiently large,

$$
\begin{aligned}
& \frac{1}{s} \int_{(0,1) \times(0, T)} \xi^{-2 s} t(T-t)\left(\left|q_{t}\right|^{2}+\left|q_{x x}\right|^{2}\right) \\
& \quad+s \int_{(0,1) \times(0, T)} \frac{\xi^{-2 s}}{t(T-t)}\left|q_{x}\right|^{2}+s^{3} \int_{(0,1) \times(0, T)} \frac{\xi^{-2 s}}{t^{3}(T-t)^{3}}|q|^{2} \\
& \leqslant C_{*}\left(\int_{(0,1) \times(0, T)} \xi^{-2 s}\left|\partial_{t} q+q_{x x}\right|^{2}+s \int_{0}^{T} \frac{\xi^{-2 s}}{t(T-t)}\left|q_{x}(1, t)\right|^{2}\right) .
\end{aligned}
$$

Let us consider now the case $b(y, t) \neq 0$. By (233),

$$
\begin{aligned}
& \frac{1}{s} \int \xi^{-2 s} t(T-t)\left(\left|q_{t}\right|^{2}+\left|q_{x x}\right|^{2}\right)+s \int \frac{\xi^{-2 s}}{t(T-t)}\left|q_{x}\right|^{2}+s^{3} \int \frac{\xi^{-2 s}}{t^{3}(T-t)^{3}}|q|^{2} \\
& \leqslant 2 C_{*}\left(\int \xi^{-2 s}\left|q_{t}+q_{x x}-b q\right|^{2}+2 \int \xi^{-2 s}|q|^{2}|b|^{2}+s \int_{0}^{T} \frac{\xi^{-2 s}}{t(T-t)}\left|q_{x}(1, t)\right|^{2}\right),
\end{aligned}
$$


or equivalently,

$$
\begin{aligned}
& \frac{1}{s} \int \xi^{-2 s} t(T-t)\left(\left|q_{t}\right|^{2}+\left|q_{x x}\right|^{2}\right) \\
& \quad+s \int \frac{\xi^{-2 s} s}{t(T-t)}\left|q_{x}\right|^{2}+s^{3} \int \xi^{-2 s}|q|^{2}\left(\frac{s^{3}}{t^{3}(T-t)^{3}}-4 C_{*}|b|^{2}\right) \\
& \leqslant 2 C_{*}\left(\int \xi^{-2 s}\left|q_{t}+q_{x x}-b q_{x}\right|^{2}+s \int_{0}^{T} \frac{\xi^{-2 s}}{t(T-t)}\left|q_{x}(1, t)\right|^{2}\right) .
\end{aligned}
$$

We take,

$$
s \geqslant C_{*}^{1 / 3} T^{2}\|b\|_{L^{\infty}((0,1) \times(0, T))}^{2 / 3}
$$

to garantee that

$$
\frac{s^{3}}{t^{3}(T-t)^{3}}-4 C_{*}|b|^{2}>0 .
$$

Redefining the constant $C_{*}$, we have that

$$
\begin{aligned}
& \frac{1}{s} \int \xi^{-2 s} t(T-t)\left(\left|q_{t}\right|^{2}+\left|q_{x x}\right|^{2}\right)+s \int \frac{\xi^{-2 s}}{t(T-t)}\left|q_{x}\right|^{2}+s^{3} \int \frac{\xi^{-2 s}}{t^{3}(T-t)^{3}}|q|^{2} \\
& \quad \leqslant C_{*}\left(\int \xi^{-2 s}\left|q_{t}+q_{x x}-b q\right|^{2}+s \int_{0}^{T} \frac{\xi^{-2 s}}{t(T-t)}\left|q_{x}(1, t)\right|^{2}\right)
\end{aligned}
$$

for all $s \geqslant s_{1}(T)\|b\|_{L^{\infty}((0,1) \times(0, T))}^{2 / 3}$, where $s_{1}(T)$ is a positive constant depending on $T$ but not on $b$.

\section{REFERENCES}

[1] M. Avellaneda, C. Bardos, J. Rauch, Contrôlabilité exacte, homogénéisation et localisation d'ondes dans un milieu non-homogène, Asymp. Anal. 5 (1992) 481-494.

[2] C. Castro, Boundary controllability of the one-dimensional wave equation with periodic oscillating density, Asymp. Anal. 20 (1999) 317-350.

[3] C. Castro, E. Zuazua, Contrôle de l'équation des ondes à densité rapidement oscillante à une dimension d'espace, C. R. Acad. Sci. Paris 324 (1997) 1237-1242.

[4] C. Castro, E. Zuazua, Low frequency asymptotic analysis of a string with rapidly oscillating density, SIAM J. Appl. Math. 60 (2000) 1205-1233.

[5] D. Cioranescu, P. Donato, An Introduction to Homogenization, Oxford Lecture Series in Mathematics and its Applications, Vol. 17, 1999.

[6] C. Fabre, J.P. Puel, E. Zuazua, Approximate controllability of the semilinear heat equation, Proc. Royal Soc. Edinburgh 125 A (1995) 31-61.

[7] H.O. Fattorini, Boundary control systems, SIAM J. Contr. 6 (1968) 349-385.

[8] H.O. Fattorini, D.L. Russell, Exact controllability theorems for linear parabolic equations in one space dimension, Arch. Rational Mech. Anal. 43 (1971) 272-292.

[9] H.O. Fattorini, D.L. Russell, Uniform bounds on biorthogonal functions for real exponentials with an application to the control theory of parabolic equations, Quart. Appl. Mat. 32 (1974) 45-69. 
[10] E. Fernández-Cara, Null controllability of the heat equation, ESAIM: COCV 2 (1997) 87103.

[11] A. Fursikov, O.Yu. Imanuvilov, Controllability of Evolution Equations, Lecture Notes Series, Vol. 34, Seoul National University, 1996.

[12] W. Krabs, On Moment Theory and Controllability of One-Dimensional Vibrating System and Heating Processes, Lecture Notes in Control and Information Sciences, Vol. 173, Springer-Verlag, 1992.

[13] G. Lebeau, The wave equation with oscillating density: observability at low frequency, ESAIM: COCV 5 (2000) 219-258.

[14] G. Lebeau, L. Robbiano, Contrôle exact de l'équation de la chaleur, Comm. PDE 20 (1995) $335-356$.

[15] G. Lebeau, E. Zuazua, Null controllability of a system of linear thermoelasticity, Archives Rat. Mech. Anal. 141 (4) (1998) 297-329.

[16] A. Lopez, Control y perturbaciones singulares de sistemas parabólicos, Ph.D. Thesis, Universidad Complutense Madrid, 1999.

[17] A. López, E. Zuazua, Uniform null-controllability for the one-dimensional heat equation with rapidly oscillating coefficients, C. R. Acad. Sci. Paris, Série I 326 (1998) 955-960.

[18] A. López, X. Zhang, E. Zuazua, Null controllability of the heat equation as singular limit of the exact controllability of dissipative wave equations, J. Math. Pures Appl. 79 (8) (2000) 741-808.

[19] D.L. Russell, A unified boundary controllability theory for hyperbolic and parabolic partial differential equations, Studies Appl. Math. 52 (1973) 189-221.

[20] L. Schwartz, Etude des sommes d'exponentielles, 2nd edition, Hermann, Paris, 1959. 